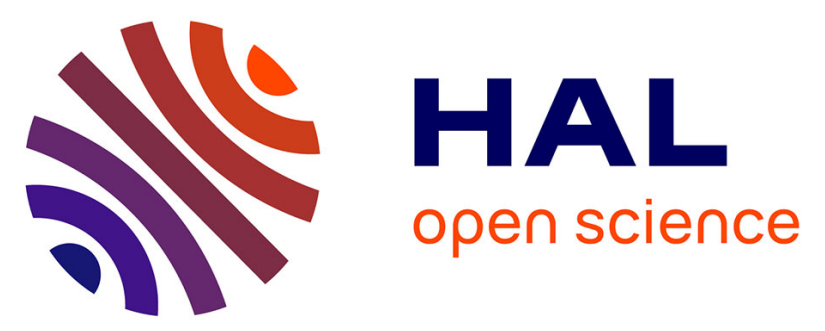

\title{
To which mixtures are French pregnant women mainly exposed? A combination of the second French total diet study with the EDEN and ELFE cohort studies
}

Thiéma Traoré, Anne Forhan, Véronique Sirot, Manik Kadawathagedara, Barbara Heude, Marion Hulin, Blandine de Lauzon-Guillain, Jérémie Botton, Marie-Aline A Charles, Amélie Crépet

\section{To cite this version:}

Thiéma Traoré, Anne Forhan, Véronique Sirot, Manik Kadawathagedara, Barbara Heude, et al.. To which mixtures are French pregnant women mainly exposed? A combination of the second French total diet study with the EDEN and ELFE cohort studies. Food and Chemical Toxicology, 2018, 111, pp.310-328. 10.1016/j.fct.2017.11.016 . hal-02906466

\section{HAL Id: hal-02906466 \\ https://hal.inrae.fr/hal-02906466}

Submitted on 24 Jul 2020

HAL is a multi-disciplinary open access archive for the deposit and dissemination of scientific research documents, whether they are published or not. The documents may come from teaching and research institutions in France or abroad, or from public or private research centers.
L'archive ouverte pluridisciplinaire HAL, est destinée au dépôt et à la diffusion de documents scientifiques de niveau recherche, publiés ou non, émanant des établissements d'enseignement et de recherche français ou étrangers, des laboratoires publics ou privés. 


\title{
To which mixtures are French pregnant women mainly exposed? A
}

\section{combination of the second French Total Diet Study with the EDEN and ELFE}

\section{cohort studies.}

T. Traoré ${ }^{1}$, A. Forhan ${ }^{2,3}$, V. Sirot ${ }^{1}$, M. Kadawathagedara ${ }^{2}$, B. Heude ${ }^{2,3}$, M. Hulin ${ }^{1}$, B. de Lauzon-Guillain ${ }^{2,3}$, J. Botton ${ }^{2,4}$, M. A. Charles ${ }^{2,3}$, A. Crépet $^{1, a}$

\footnotetext{
${ }^{1}$ ANSES, French Agency for Food, Environmental and Occupational Health \& Safety, 14 rue Pierre et Marie Curie, 94701 Maisons-Alfort, France.

2 INSERM, UMR1153 Epidemiology and Biostatistics Sorbonne Paris Cité Center (CRESS), Early Origin of Child Health and Development Team (ORCHAD), Paris, F-75014 France.

${ }^{3}$ Paris Descartes University, France.

${ }^{4}$ Faculty of Pharmacy, Paris-Sud University, Paris-Saclay University, 92296 Châtenay-Malabry, France.
}

\begin{abstract}
Pregnant women and their unborn child are exposed to a large number of substances during pregnancy. Some of these substances may cross the placenta, resulting in exposure of the foetus. There is growing evidence that certain substances could interact to produce a mixture effect. It is therefore essential to identify the main mixtures mothers are exposed to.

This study aimed to identify the major mixtures French pregnant women included in EDEN and ELFE cohorts were exposed to, on the basis of the 441 substances analysed in the second French total diet study. Exposure systems and the composition of substances were identified from co-
\end{abstract}

\footnotetext{
Abbreviations

BMI, body mass index; EDEN, study on the pre- and early postnatal determinants of child health and development; ELFE, French longitudinal study since childhood; FFQ, food frequency questionnaire; INCA, Individual and National Food Consumption Survey; LB, lower bound; SNMU, sparse non-negative matrix underapproximation; TDS, Total Diet Study

${ }^{a}$ Corresponding author: A. Crépet; email address: amelie.crepet@anses.fr
} 
exposures using sparse non-negative matrix under-approximation to generate the main mixtures. Individuals were clustered to define clusters with similar co-exposure profiles.

Six clusters associated with eight mixtures were identified. For example in ELFE, cluster 2 comprising $10 \%$ of the population was characterised by mixtures "Pest- 1 " mainly contains pesticides and "TE-F-PAH" contains trace elements, furans and polycyclic aromatic hydrocarbons. Five other clusters were also described with their associated mixtures. Similar results were observed for EDEN.

This study helps to prioritise mixtures for which it is crucial to investigate possible toxicological effects and to recommend epidemiological studies concerning health effects.

\section{Keywords}

Co-exposure assessment, sparse non-negative matrix under-approximation, chemical mixtures, pregnant women, individual clusters.

\section{Highlights}

- Exposures to 200 substances were assessed for French women before and during their pregnancy

- Co-exposure data was reduced into two matrices using SNMU method: exposure systems and individuals' coefficients

- Eight main mixtures were defined from exposure systems and six of them were common

- Six clusters of mothers with similar pattern of contaminant intake were identified

\section{Introduction}

Populations worldwide are exposed to a wide range of chemicals through their diet, and pregnant women are no exception. The foetal and early postnatal periods are considered to involve increased susceptibility to long-term effects (Sly and Flack, 2008). Numerous studies have shown associations between prenatal exposure to environmental contaminants that cross the placental barrier, in particular endocrine disruptor chemicals, and postnatal growth (Mendez et al., 2011; Valvi 
et al., 2012) or between acrylamide exposure and prenatal growth (Pedersen et al., 2012; DuarteSalles et al., 2013). Heavy metals have also been found to have an impact, in particular on cognitive, motor and intellectual development (Canfield et al., 2003; Dorea and Donangelo, 2006).

In most cases, chemicals are studied individually and the potential interactions or additive effects of substances are not taken into account. However, some recent epidemiological studies have highlighted relationships between exposure to mixtures of substances and health effects. For example, Czarnota et al. (2015) showed an association between exposure to a mixture of five polychlorinated biphenyls (PCBs), seven polycyclic aromatic hydrocarbons (PAHs) and 15 pesticides and the risk of non-Hodgkin lymphoma. On the basis of a literature review, Claus Henn et al. (2014) noted several interactions between substances and health effects in children. For example, an interaction between cadmium and lead on reproductive hormones and neurodevelopment was highlighted. The authors also emphasised the need to improve statistical tools to study exposure to mixtures. It is still a challenge to determine which substances should be studied together. For example, EFSA (2013) proposed cumulative assessment groups (CAGs) based on grouping pesticides by their organ toxicity or specific effects. However, these CAGs do not take into account the probability of co-exposure of an individual to substances in a particular CAG. Over the last seven years, methods have been proposed to identify major mixtures from environmental exposures. Given the high number of substances to which populations are exposed, it is necessary to develop adapted statistical methods to reduce the dimension, to consider and to identify mixtures. Crépet and Tressou (2011) used a Bayesian non-parametric model to determine major pesticide mixtures. More recently, an approach based on non-negative matrix factorisation (NMF) (Lee and Seung, 2001) and clustering methods was used to identify the primary mixtures associated with specific diets (Béchaux et al., 2013; Traoré et al., 2016). The NMF and clustering methods were used to define dietary patterns and clusters of individuals with similar diets (Zetlaoui et al., 2011; Sy et al., 2013; Gazan et al., 2016). 
The objective of this study was to describe the main mixtures pregnant women in France are exposed to, using a modified version of the NMF method, called sparse non-negative matrix underapproximation (SNMU) (Gillis and Plemmons, 2013). Exposures to up to 200 substances were estimated from two datasets: firstly, the dietary consumption of pregnant women in France before and during pregnancy on the basis of the EDEN and ELFE mother-child cohorts; secondly, substance concentration levels in foods provided by the second French Total Diet Study (TDS 2). The SNMU was completed by hierarchical clustering to classify groups with similar exposure profiles. Mixtures identified from the various exposure data are described in this paper for both cohorts. The exposure profiles are described only for ELFE cohort.

\section{Materials and methods}

\subsection{Food Consumption}

Pregnant women before 24 weeks of amenorrhea $(N=2,002)$ aged from 18 to 45 years were included in the EDEN mother-child cohort. Recruitment took place between February 2003 and January 2006 in two university hospitals in France in the cities of Nancy and Poitiers (Heude et al., 2016). Participants completed a validated (Deschamps et al., 2009) food frequency questionnaire (FFQ) at two time points. The first FFQ was recorded at inclusion and corresponds to maternal diet in the year before pregnancy. The second FFQ was completed at delivery and concerned maternal diet during the last trimester of pregnancy. The consumption frequency of 137 food and beverage items was collected with a 7-item scale from "never" to "more than once a day". Portion sizes were determined using pictures for 12 food types (meat, chips, pasta, vegetables, cakes, cheese) on a three-level scale and for other food types, sizes were standard portions for the adult population in France (Hercberg et al., 2002). Individual daily intake of each food item was assessed by combining the frequency of intake and portion size. Subjects for whom more than three food items of the FFQ were missing were excluded. For the subjects with only one or two missing items, the median values 
of daily intakes were attributed. In all, 1,806 FFQs for the diet before pregnancy and 1,666 for the diet during the last trimester of pregnancy were included in this analysis.

The ELFE cohort study is another mother-child investigation on a larger and highly representative sample of pregnant women in mainland France in 2011 (Vandentorren et al., 2009). Women attending 320 randomly selected maternity hospitals were asked to participate. Recruitment was carried out in four waves of 4 to 8 days. An FFQ modified from the EDEN FFQ was used to collect data on dietary habits for 18,042 women during the last trimester of pregnancy. The consumption frequency of 125 food and beverage items was collected using a 7-item scale from "never" to "more than once a day". The portion sizes of 75 food items were estimated using photos for different food types on a 5-level scale based on the SU.VI.MAX portion book (Hercberg, 2000). Individual daily intake of each food item was assessed by combining frequency of intake and portion size. Individuals with more than 10 missing food items in their FFQ were excluded. For the subjects with fewer than ten missing items, the median values of daily intakes were attributed. Finally, intake data were available for 15,226 women.

\subsection{Concentration data}

The French total diet study (TDS 2) (Sirot et al., 2009) provided concentration levels for 441 substances in 212 core foods (Arnich et al., 2012; Bemrah et al., 2012; Nougadère et al., 2012; Sirot et al., 2012a; Sirot et al., 2012b; Sirot et al., 2013; Veyrand et al., 2013; Bemrah et al., 2014; Rivière et al., 2014). The 212 core foods cover about $90 \%$ of the whole diet of the French population, as estimated from the second French national food consumption survey (INCA 2) (Dubuisson et al., 2010; Lioret et al., 2010). In order to be representative of consumer habits in France, each type of food considered was composed of 15 samples of the same food, so as to cover different varieties, purchase locations, preparation methods, cooking methods, etc. In all, 19,785 food products were purchased in eight regions in France over different seasons from 2007 to 2009 to make up the 1,319 composite samples of core foods to be analysed for additives, environmental contaminants, pesticide residues, trace elements and minerals, mycotoxins, phytoestrogens and acrylamide. 
As recommended in GEMS/Food-EURO (2013), non-detected values were replaced by 0 and detected values unable to be quantified, by the limit of detection. With this scenario, called the lower bound (LB) scenario, the exposure of the whole population is equal to zero for 210 pesticides, four perfluoroalkyl acids and seven mycotoxins that were not considered in this study. In addition, 10 minerals ( $\mathrm{Ca}, \mathrm{Cu}, \mathrm{Fe}, \mathrm{K}, \mathrm{Mg}, \mathrm{Mn}, \mathrm{Mo}, \mathrm{Na}$, Se and $\mathrm{Zn}$ ) out of 13 analysed in TDS 2 were not considered in the present study, in the absence of potential adverse effects on development or health. The remaining 210 out of 441 substances were considered, including:

> 21 trace elements and minerals: aluminium ( $\mathrm{Al}$ ), antimony ( $\mathrm{Sb})$, barium (Ba), cadmium (Cd), cobalt (Co), gallium $(\mathrm{Ga})$, germanium $(\mathrm{Ge})$, lithium (Li), lead $(\mathrm{Pb})$, nickel $(\mathrm{Ni})$, silver $(\mathrm{Ag})$, strontium $(\mathrm{Sr})$, tellurium $(\mathrm{Te})$, tin $(\mathrm{Sn})$ and vanadium (V). Separate analyses were performed to take into account the proportion of inorganic and organic arsenic (As) and mercury ( $\mathrm{Hg})$, and the proportion of trivalent and hexavalent chromium $(\mathrm{Cr})$. Inorganic and organic arsenic (Asi and Aso) and mercury ( $\mathrm{Hgl}$ and $\mathrm{MeHg}$ ) and trivalent and hexavalent chromium (Crlll and $\mathrm{CrVI}$ ) were therefore considered instead of total arsenic, chromium and mercury;

$>17$ congeners of polychlorinated dibenzo-p-dioxins (or dioxins) and polychlorinated dibenzofurans (or furans) (PCCD/F) (HCDD/F-123478, HCDD/F-123678, HCDD/F-123789, HCDD/F-1234678, OCDD/F, PCDD/F-12378, TCDD/F-2378, HCDF-1234789, HCDF-234678, PCDF-23478);

> 12 congeners of 'dioxin-like' polychlorinated biphenyls (PCBs) (PCB-77, 81, 105, 114, 118, 123, 126, 156, 157, $167,169,189)$;

$>$ six congeners of 'non dioxin-like' polychlorinated biphenyls (PCB-28, 52, 101, 138, 153, 180);

$>12$ perfluoroalkyl acids (PFAAs): nine carboxylates (PFDA, PFDoA, PFHpA, PFHxA, PFNA, PFOA, PFTeDA, PFTrDA, PFUnA) and three sulfonates (PFBS, PFHXS, PFOS);

> 14 brominated flame retardants (BFRs): eigth polybrominated diphenyl ether congeners (PBDE-28, 47, 99, 100, 153, 154, 183, 209), three polybrominated biphenyl congeners (PBB-52, 11, 153) and three hexabromocyclododecane congeners (HBCD-alpha, beta, gamma);

> 18 mycotoxins: fumonisins $\mathrm{B} 1$ and $\mathrm{B} 2$ (FB1, FB2), ochratoxin A, B (OTA, OTB) and patulin (Pat), trichothecenes from group A including T2-toxin, HT2-toxin, diacetoxyscirpenol (DAS) and monoacetoxyscirpenol (MAS); group B including nivalenol (NIV), deoxynivalenol (DON), de-epoxy derivative of DON (DOM-1), 3-acetyldeoxynivalenol (DON3), 15-acetyldeoxynivalenol (DON15), and fusarenon X (FusX), zearalenone (Zea) and its metabolites: alphazearalanol and alpha-zearalenol; 
> 11 phytoestrogens: biochanin A, coumestrol, daidzein, enterolactone, equol, , formononetin, genistein, glycitein, matairesinol, resveratrol, and secoisolariciresinol;

$>73$ active pesticide residues;

> 4 additives: nitrites, sulphites, tartaric acid and annatto;

> 21 heat-induced contaminants including acrylamide and 20 congeners of polycyclic aromatic hydrocarbons (PAHs): anthrancene (AN), benzo[a]anthracene (BaA), benzo[a]pyrene (BaP), benzo[b]fluoranthene (BbF), benzo[c]fluorine (BcFL), benzo[g,h,i]perylene (BghiP), benzo[j]fluoranthene (BjF), benzo[k]fluoranthene (BkF), chrysene (CHR), cyclopenta(c,d)pyrene (CPP), dibenzo[a,h]anthracene (DBahA), dibenzo[a,e]pyrene (DbaeP), dibenzo[a,h]pyrene (DbahP), dibenzo[a,i]pyrene (DbaiP), dibenzo[a,I]pyrene (DbalP), fluoranthene (FA), indeno[1,2,3-cd]pyrene (IP), 5-methylchrysene (MCH), phenanthrene (PHE) and pyrene (PY);

$>\quad$ and bisphenol A (BPA)

\subsection{Matching the TDS 2 with FFQ data from the EDEN and ELFE cohort studies}

Since some differences were observed between food items from TDS 2 and the two cohorts, matching between the nomenclatures of these studies was conducted. In most cases (65\% for EDEN and $55 \%$ for ELFE), a food item from the two cohorts was comparable to a food item from TDS 2 . In other cases, different scenarios were used:

$>$ Correlation scenario: the food items from the two cohorts were more detailed than in TDS 2. For example, in the two cohorts, carrots are separated into "cooked carrot" and "raw carrot", while in TDS 2 the food item "carrots" covers cooked and raw carrots. In this case, the concentration of the TDS 2 food item "carrots" was used for both detailed food items in the cohorts.

$>$ Grouping scenario: a cohort food item is covered by several food items from TDS 2. In this case, the weighted mean of the concentrations of related TDS 2 food items was attributed to these cohorts' food items. The weights were estimated by the ratios of consumed quantity of each food item by the female population aged 18 to 45 years of the INCA 2 study. This scenario was applied for example to the category "dried vegetables" in the two cohorts and equivalent to "white bean" + "lentil" in TDS 2.

$>$ Recipes scenario: this case concerns only the category considered as composite foods in the two cohorts. For these products, the concentrations were calculated by using recipes. For example, it is applied to the "gratin dauphinois" equivalent to "potatoes" + "semi-skimmed milk" + "eggs" + "butter" + "cream" in TDS 2. 
After the matching process, the five food items in EDEN (avocado, pumpkin, prepared light meals, sweetener and whisky) and the six in ELFE (avocado, pumpkin, mushroom, other starches, prepared light meals and exotic fruits) with no TDS 2 correspondence were excluded. Finally, 207 substances were associated with 132 food items in EDEN, and 210 substances with 119 food items in ELFE.

\subsection{Exposure assessment}

Exposure $e_{n p}$ of woman $n$ to contaminant $p$ was assessed (see equation below) by combining the concentration $c_{f p}$ in food $f$ of contaminant $p$ with the average daily intake $q_{n f}$ of woman $n$ to food $f$ and summing over all the food items reported as consumed by each woman.

$$
e_{n p}=\sum_{f=1}^{F} q_{n f} \times c_{f p}
$$

The exposure of the population of $N$ individuals to $P$ substances can be expressed as a matrix $E$ of dimension $(P \times N)$.

Three different exposure matrices were obtained: two in the EDEN cohort (i) before pregnancy (EDEN before pregnancy) and (ii) at the end of pregnancy (EDEN during pregnancy), and (iii) one in the ELFE cohort at the end of pregnancy (ELFE during pregnancy). To deal with differences of variability between the exposures, each exposure matrix was standardised, i.e. $E$ was multiplied by the $(P \times P)$ inverse diagonal matrix of the standard deviations of the exposure to each substance across individuals $S_{E}: E^{*}=S_{E}^{-1} E$.

\subsection{Mixture identification}

\subsubsection{NMF with under-approximation and sparsity constraints: the SNMU}

Non-negative matrix factorisation (NMF) (Lee and Seung, 2001) consists in factorising a nonnegative matrix by the product of two low-rank nonnegative matrices. The NMF is performed using an optimisation method with a non-negativity constraint. Applied to the $(P \times N)$ exposure matrix $E$ and for a given number of factorial dimensions $K$ designating the number of exposure systems, the 
optimal approximation $W$ and $H$ of dimensions $(P \times K)$ and $(K \times N)$, respectively was obtained by minimising the following equation:

$$
\min _{W \in \mathbb{R}^{P \times K}, H \in \mathbb{R}^{K \times N}}\|E-W H\|_{F}^{2} \text { such that } W \geq 0 \text { and } H \geq 0 \text {. }
$$

In the exposure systems matrix $W$, each column represents an exposure system and each element $w_{p k}$ gives the contribution of the substance $p$ in the exposure system $k$. The matrix $H$ defines the individuals' coefficient matrix and each element $h_{k n}$ gives the contribution of the exposure system $k$ to an individual's overall exposure $n$. The NMF equation was solved by a multiplicative algorithm based on a gradient descent approach (Lee and Seung, 2001) and exposure systems were extracted at the same time.

Recently, Gillis and Glineur (2010) introduced a new approach called non-negative matrix under-approximation (NMU) and proposed a recursive algorithm based on Lagrangian relaxation to solve the NMF equation. The principle of the recursive algorithm was to find exposure systems one by one from the exposure matrix $E$. To ensure non-negativity, Gillis and Glineur (2010) added a new constraint to the optimisation process: $W H \leq E$ i.e. each exposure $e_{p n}$ must be greater than its approximation $w_{p k} h_{k n}$.

As substances can, in some cases, have small coefficients relative to the other system contributors, Gillis and Plemmons (2013) proposed adding a sparsity constraint to enforce separation of the lowest contributors using a penalty $\mu \geq 0$ on the exposure matrix $W$. Thus, the new model, called sparse NMU (SNMU), is defined by the following optimisation problem (Gillis and Plemmons, 2013):

$$
\min _{W \in \mathbb{R}^{P \times K}, H \in \mathbb{R}^{K \times N}}\|E-W H\|_{F}^{2}+\mu\|W\|_{0} \text { such that } W \geq 0, H \geq 0 \text { and } W H \leq E,
$$

Finally, to define the mixture from an exposure system, substances were ordered by decreasing contribution to the exposure system expressed in percentage (\%Subst in Tables 1, 2, 3 and 4). For practical purposes, it was chosen to present the first 25 substances in each mixture. 
The SNMU was recoded on R (Team, 2016) software using the implementation algorithm on Matlab software by Gillis and Plemmons (2013). The original code is available on their website: https://sites.google.com/site/nicolasgillis/code.

\subsubsection{Hierarchical clustering method}

Following the SNMU, a clustering method was applied to identify clusters of women with similar co-exposure profiles. Hierarchical agglomerative clustering was applied, using the hclust function in $\mathrm{R}$ (Team, 2016) software, on the matrix $H$ containing the contributions of each exposure system the individuals' exposures. The Euclidian distance between individuals and the Ward aggregation criterion were used as parameters in the hclust function. The principle of the hclust method is to start with $N$ clusters: every individual $n$ represents a cluster. In the second step, two clusters are grouped into a bigger one, on the basis of their distance. After step $N-1$, a single cluster composed of all the individuals is obtained. Given that at each level of aggregation, some inter-class inertia is lost, the optimal number of clusters is that preceding a significant loss of inertia.

The catdes function from the FactoMineR package (Lê et al., 2008) was used to determine the principal exposure systems characterising each cluster. It was also used to compare the mean of characteristics (age, BMI before pregnancy and weight gain) and exposure of the individuals from the different clusters with those of the overall population. The $p$-value from the F-test in one-way analysis of variance of the catdes function was compared with the standard $p$-value 0.05 to indicate a significant difference between individuals in a cluster and the whole population.

\subsubsection{Selection of the number $\boldsymbol{K}$ of exposure systems and sparsity parameter}

The determination of the optimal number of exposure systems $K$ remains a challenge with NMF. In their studies, Zetlaoui et al. (2011), Sy et al. (2013) and Béchaux et al. (2013) used a criterion based on the residual sum of squares between the exposure and the estimated matrices. With the large number of substances, this criterion does not produce results allowing an optimal choice of $K$ but gives indications on candidate values to the optimal choice. In their studies, Gazan et al. (2016) and Traoré et al. (2016) supplemented the residual sum of squares criterion using their indications by 
the relevance and quality of interpretation of clustering results. In this study, the residual sum of squares criterion was used initially and consists in applying the SNMU on exposure matrix $E$ with a set of values $K$ and of plotting the residual sum of squares for each value $K$. The criterion based on the relevance and quality of interpretation consisted in using the candidate values obtained from the residual sum of squares criterion and in examining how the exposure systems characterise the clusters. The $K$ values for which one or more exposure systems were not used to characterise a cluster were rejected.

As proposed by Gillis and Plemmons (2013), the SNMU requires human supervision to adjust the penalty parameter $\mu$. This parameter was applied to enforce sparsity on each column of the exposure matrix $W$. Gillis and Plemmons (2013) introduced a parameter $\lambda \in\left[0,1{ }^{k}\right.$ and each $\lambda_{k}$ is proportional to the penalty parameter. A lower and upper bound, respectively $\delta$ and $\Delta$, were imposed on the sparsity $\lambda_{k}$ of each column of the exposure systems matrix $W$ : new parameters were then defined $0 \leq \delta<\Delta \leq 1$. The value of parameter $\lambda_{k}$ is decreased (resp. increased) when the lower (resp. upper) bound is reached for a given iteration (Gillis and Plemmons, 2013). The SNMU was applied to the exposure data $E$ and several values of the parameters $\lambda, \delta$ and $\Delta$ were tested regarding their effect on the mixture weights.

\section{Results}

\subsection{The number $K$ of exposure systems and the sparsity coefficient}

The residual sum of squares criterion was first applied to exposure data $E$ from EDEN before pregnancy and candidate values to the optimal choice of $K$ were around $K=8$. Secondly, the criterion based on the relevance and quality of interpretation was applied with values $K=7,8,9$ and 10 . With 7 and 8 , each exposure characterises at least one cluster, whereas with 9 and 10 one or two exposure systems were unused to characterise a cluster. Thus, the optimal choice of the number $K$ of exposure systems was set to 8 . 
For each $k$, the lower bound $\delta$ and the upper bound $\Delta$ were set to 0.2 and 0.99 . The selected values for $\lambda$ were $0.95,0.8,0.65,0.5,0.4,0.4,0.4$ and 0.4 for the different $k$ values.

\subsection{EDEN and ELFE mixtures}

The mixtures obtained on the basis of data from EDEN for before (Table 1) and during pregnancy (Table 2) and those from ELFE data (Table 3) were very similar. Overall, six mixtures were common to the three datasets. The tables 1 to 3 present the contribution (\%) of each substance to the mixture composed of 25 substances. The total contribution of the 25 substances per exposure system is also presented.

\subsubsection{Common mixtures}

The "TE-F-PAH" mixture, identified from EDEN exposure data before pregnancy and composed mostly of trace elements and furans, was also identified from EDEN and ELFE exposure data during pregnancy. The substances common to each mixture were composed mostly of 11 trace elements (inorganic arsenic, chromium VI, vanadium, lead, tellurium, chromium III, cobalt, nickel, cadmium, barium and aluminium), five furans (OCDF, HCDF-234678, 1234789, 123478 and 1234678) and the PAH pyrene. In EDEN before pregnancy, these substances were also associated with two other trace elements (germanium and strontium), five PAHs (benzo[g,h,i]perylene, indeno[1,2,3-cd]pyrene, cyclopenta(c,d)pyrene, benzo[a]pyrene and fluoranthene) and PFOA. In EDEN during pregnancy, they were also associated with trace elements (germanium and strontium), PAHs (benzo[g,h,i]perylene and fluoranthene), dioxins (HCCD-123678 and 1234678), furan (HCDF-123678) and PFOA. In ELFE, they were also associated with trace elements (antimony and lithium), dioxins (HCDD-123678, 1234678 and 234678), furans (HCDF-123678 and 123789) and zearalenone.

The "PCB-BFR-Aso-MeHg" mixture identified from EDEN before pregnancy was also identified from EDEN and ELFE during pregnancy. For the common substances, the mixtures were composed of three NDL-PCBs (PCB-101, 153 and 180), three DL-PCBs (PCB-156, 167 and 189), seven BFRs (PBDE-28, 47, 100, and 154; PBB-52, 101 and 153) and two trace elements (organic arsenic and methyl mercury). In EDEN before pregnancy, the mixture also contained NDL-PCB 138, five DL-PCBs (PCB-77, 105, 123, 
157, and 169), three PFAAs (PFOS, PFUnA and PFTrDA) and a furan (TCDF 2378). In EDEN during pregnancy, the mixture also contained three PFAAs (PFUnA, PFTrDA and PFOS), NDL-PCB 28, two DLPCBs (PCB-81 and 123), two BFRs (PBDE-99 and 153), a furan (PCDF-12378) and inorganic mercury. In ELFE during pregnancy, the mixture also contained six DL-PCBs (PCB-77, 105, 114, 118, 123 and 157), two NDL-PCBs (PCB-52 and 138) and two furans (PCDF-12378 and TCDF-2378).

The "Pest-1" mixture composed mainly of pesticides and identified from EDEN before pregnancy was also identified from EDEN and ELFE during pregnancy. It was composed of four carbamates (carbendazim, thiabendazole, pirimicard and methomyl), four organophosphates (phosmet, phosalone, azinphos-methyl and chlorpyrifos-ethyl), two dicarboximides (captan and folpet), three benzoylureas (diflubenzuron, triflumuron and teflubenzuron), seven other pesticides (propargite, ethoxyquin, diphenylamine, tebufenozide, fludioxonil, boscalid and bifenthrin) and a mycotoxin (patulin). This mixture was also associated with four pesticides (pyrimethanil, cyprodinyl, imazalil and iprodione) in EDEN before pregnancy and four pesticides (pyrimethanil, phenylphenol, lambdacyhalothrin and cyprodinyl) in ELFE, whereas in EDEN during pregnancy, it was also associated with two pesticides (ethion and imazalil), a PFAA (PFNA) and a phytoestrogen (daidzein).

In addition, the "Pest-2" mixture identified from EDEN before pregnancy and composed mostly of pesticides was also identified from EDEN and ELFE during pregnancy. It was composed of two pyrethroids (acrinathrin and lambda-cyhalothrin), two strobilurns (kresoxim-methyl and azoxystrobin), three organophosphates (dichlorvos, dimethoate and chlorpyrifos-ethyl), three triazoles (penconazole, tebuconazole and fenbuconazole), two dicarboximides (procymidone and iprodione) and seven other pesticides (endosulfan, bupirimate, mepanipyrim, fenhexamid, cyprodinyl, metalaxyl and boscalid). This mixture was also associated with six pesticides (myclobutanil, pyrimethanil, tetradifon, pyriproxyfen, diethofencarb and chlorothalonil) in EDEN before pregnancy, three pesticides (fludioxonyl, phosmet and chlorothalonil), two trace elements (nickel and silver) and an additive (sulphites) in EDEN during pregnancy. In ELFE, "Pest-2" was also 
associated with four pesticides (phosmet, fludioxonil, chlorthal dimethyl and pyriproxyfen), a trace element (nickel) and an additive (sulphites).

The mixture "Pest-3" identified from EDEN before pregnancy and composed mainly of pesticides, was also identified from EDEN and ELFE during pregnancy. It was composed of two pyrethroids (cyfluthrin and bifenthrin), three triazoles (tetraconazole, triadimenol and myclobutanil), two carbamates (methomyl and metalaxyl) and nine other pesticides (trifloxystrobin, spiroxamine, quinoxyfen, etofenprox, tebufenpyrad, pyrimethanil, fenhexamid, teflubenzuron and cyprodinyl). In EDEN before pregnancy, this mixture was also associated with four PFAAs (PFHxA, PFBS, PFHxS and PFHpA) and five pesticides (cyproconazole, sulphur, pyriproxyfen, diethofencarb and chlorothalonil). Eight pesticides (mepanipyrim, penconazole, boscalid, chlorpyrifos-methyl, chlorpyrifos-ethyl, iprodione, azoxystrobin and lambda-cyhalothrin) and a trace element (silver) were also associated with this mixture in EDEN during pregnancy. In ELFE, nine pesticides (mepanipyrim, penconazole, boscalid, iprodione, chlorpyrifos-ethyl, lambda-cyhalothrin, procymidone, azoxystrobin and chlorpyrifos-methyl) were also associated with this mixture.

The main substances identified in the "PFAA-Ge-Li" mixture from EDEN before pregnancy were also identified from EDEN and ELFE during pregnancy. This mixture was composed of five PFAAs (PFHXS, PFBS, PFHxA, PFHpA and PFOA) and two trace elements (germanium and lithium). In EDEN before pregnancy, this mixture was also associated with seven pesticides (pyriproxyfen, tetradifon, sulphur, diethofencarb, chlorothalonil, cyproconazole and bifenthrin), six other trace elements (strontium, vanadium, inorganic arsenic, gallium, inorganic mercury and antimony), three other PFAAs (PFOS, PFTeDA and PFDoA), a PAH (dibenzo[a,h]pyrene) and a mycotoxin (patulin). In EDEN during pregnancy, this mixture also contained six other trace elements (strontium, inorganic arsenic, vanadium, chromium $\mathrm{VI}$, inorganic mercury and tellurium), six phytoestrogens (formononetin, equol, enterolactone, matairesinol, glycitein and resveratrol), a PAH (5-methylchrysene), two mycotoxins (DON15 and FB2) and three pesticides (malathion, phenylphenol and carbaryl). In ELFE, this mixture was also associated with nine other pesticides (carbendazim, phenylphenol, pyriproxyfen, tetradifon, 
lindane, sulphur, carbofuran, diethofencarb and chlorothalonil), three other trace elements (chromium VI, tin and gallium), four mycotoxins (FB1, OTA, FB2 and DON15), a PFAAs (PFOS) and a phytoestrogen (equol).

\subsubsection{Other Mixtures}

Five mixtures were found in only one or two datasets.

The "Myco-Pest-PAH" mixture identified from EDEN before pregnancy was also found in EDEN during pregnancy. It contained eight mycotoxins (alpha-zearalanol, alpha-zearalenol, DAS, DON3, FusX, OTB, OTA and HT2-toxin), three pesticides (chlorpyrifos-methyl, cyproconazole and pirimiphosmethyl) and four PAHs (benzo[g,h,i]perylene, benzo[e]pyrene, cyclopenta(c,d)pyrene and indeno[1,2,3-cd]pyrene). In EDEN before pregnancy, these substances were associated with nine other pesticides (pyriproxyfen, tetradifon, sulphur, chlorothalonil, diethofencarb, flutriafol, iprodione, ethion and bifenthrin) and an additive (sulphites). In EDEN during pregnancy, these substances were associated with three other mycotoxins (DON, DON15 and zearalenone), a PAH (pyrene), two phytoestrogens (daidzein and genistein), a trace element (gallium), a pesticide (sulphur) and two PFAAs (PFBS and PFHxS).

The "Mixt-1" mixture from EDEN before pregnancy was composed of acrylamide, seven mycotoxins (T-2 toxin, nivalenol, OTB, FB1, MAS, FB2, and DON15), six PAHs (dibenzo[a,i]pyrene, anthracene, benzo[c]fluorine, 5-methylchrysene, dibenzo[a,I]pyrene and benzo[k]fluoranthene), four pesticides (chlorpropham, hexachlorobenzene, lindane and phenylphenol), but also some additives (nitrites and tartaric acid), phytoestrogens (glycitein, enterolactone, equol and formononetin) and a trace element (tin).

The "Mixt-2" mixture from EDEN during pregnancy was composed of nine DL-PCBs (PCB-77, 105, 114, 118, 126, 156, 157, 167 and 169), three NDL-PCBs (PCB-52, 138 and 153), two BFRs (HBCD alpha and PBDE-47), seven PAHs (chrysene, benzo[a]anthracene, benzo[j]fluoranthenen, benzo[b]fluoranthene, benzo[k]Fluoranthene, benzo[c]fluorine and bibenzo[a,e]pyrene), inorganic mercury, a furan (TCDF2378), bisphenol $A$ and $T 2$ toxin. 
Two other mixtures were identified from ELFE during pregnancy only and were composed of heterogeneous substances: the "Mixt-3" and "Mixt-4" mixtures. The "Mixt-3" mixture was composed of PFAAs (PFTeDA, PFDoA, PFDA, PFOS and PFNA), fourteen PAHs (benzo[k]fluoranthene, benzo[b]fluoranthene, benzo[j]fluoranthene, dibenzo[a,i]pyrene, chrysene, benzo[a]anthracene, dibenzo[a,e]pyrene, benzo[a]pyrene, indeno[1,2,3-cd]pyrene, dibenzo[a,h]anthracene, dibenzo[a,I]pyrene, benzo[c]fluorene, benzo[g,h,i]perylene and fluoranthene), trace elements (silver, organic arsenic and cadmium), BFRs (HBCD beta and gamma) and dioxin OCDD. The "Mixt-4" mixture contained mostly pesticides with among others chlorothalonil, three carbamates (diethofencarb, carbofuran and metalaxyl), two dicarboximides (procymidone and iprodione), two pyrethroids (bifenthrin and lambda-cyhalothrin) and three triazoles (cyproconazole, flutriafol and tebuconazole). "Mixt-4" also contained trace elements (tin and lithium) and a phytoestrogen (secoisolariciresinol). 


\begin{tabular}{|c|c|c|c|c|c|c|c|c|c|c|c|c|c|c|c|}
\hline \multicolumn{16}{|c|}{ EDEN mixtures before pregnancy } \\
\hline TE-F-PAH (30.5\%) & \% Subst & \begin{tabular}{|c|} 
PCB-BFR-Aso-MeHg \\
$(11.3 \%)$
\end{tabular} & \% Subst & Pest-1 (8.6\%) & \% Subst & Mixt-1 (15.8\%) & \% Subst & Pest-2 (8.4\%) & \% Subst & Pest-3 (7.6\%) & \% Subst & PFAA-Ge-Li (11.6\%) & \% Subst & Myco-Pest-PAH (6.2\%) & \% Subst \\
\hline Inorganic arsenic & 5.51 & PCB 153 & 4.54 & Carbendazim & 5.26 & Acrylamide & 7.10 & Acrinathrin & 6.83 & Cyfluthrin & 9.20 & PFHXS & 17.1 & Alpha-zearalanol & 15.1 \\
\hline Germanium & 5.37 & PCB 157 & 4.51 & Thiabendazole & 4.91 & $T-2$ toxin & 6.52 & Endosulfan & 6.67 & Tetraconazole & 9.20 & PFBS & 17.1 & Alpha-zearalenol & 15.1 \\
\hline Chromium VI & 5.26 & PCB 138 & 4.50 & Propargite & 4.89 & Nivalenol & 6.16 & Bupirimate & 6.65 & Trifloxystrobin & 9.20 & PFHXA & 16.7 & DAS & 15.1 \\
\hline Strontium & 4.83 & PCB 167 & 4.49 & Phosmet & 4.84 & Dibenzo[a,i]pyrene & 5.52 & Kresoxim-methyl & 6.64 & Spiroxamine & 9.20 & PFHpA & 16.4 & DON3 & 15.1 \\
\hline Vanadium & 4.55 & PCB 156 & 4.43 & Pirimicarb & 4.79 & Anthracene & 5.46 & Dichlorvos & 6.62 & Quinoxyfen & 9.20 & PFOA & 7.85 & Fus X & 15.1 \\
\hline Lead & 4.42 & PCB 123 & 4.43 & Ethoxyquin & 4.74 & ОТВ & 5.26 & Penconazole & 6.43 & Etofenprox & 9.20 & Strontium & 5.91 & ОТВ & 2.77 \\
\hline Tellurium & 4.35 & PBDE 47 & 4.41 & Phosalone & 4.72 & FB1 & 5.24 & Mepanipyrim & 6.19 & Tebufenpyrad & 9.20 & Germanium & 4.68 & OTA & 2.33 \\
\hline Chromium III & 4.19 & PCB 180 & 4.35 & Diphenylamine & 4.72 & Chlorpropham & 4.93 & Azoxystrobin & 5.61 & Triadimenol & 9.14 & Lithium & 2.64 & Chlorpyrifos-methyl & 1.95 \\
\hline Cobalt & 4.17 & PCB 189 & 4.31 & Azinphos-methyl & 4.71 & Nitrites & 4.90 & Fenhexamid & 4.77 & Myclobutanil & 4.11 & Pyriproxyfen & 1.09 & Benzo[g,h,i]perylene & 1.94 \\
\hline Nickel & 3.87 & PCB 101 & 4.24 & Captan & 4.71 & Glycitein & 4.68 & Tebuconazole & 4.61 & Pyrimethanil & 3.54 & Tetradifon & 1.06 & Benzo[e]pyrene & 1.76 \\
\hline Cadmium & 3.78 & PBDE 100 & 4.14 & Diflubenzuron & 4.71 & MAS & 4.18 & Dimethoate & 4.60 & Fenhexamid & 3.36 & Sulphur & 1.04 & Pyriproxyfen & 1.39 \\
\hline Benzo[g,h,i]perylene & 3.71 & PBDE 154 & 4.12 & Tebufenozide & 4.71 & Benzo[c]fluorene & 4.13 & Fenbuconazole & 4.57 & Bifenthrin & 2.65 & Diethofencarb & 0.97 & Tetradifon & 1.33 \\
\hline Barium & 3.69 & PBDE 28 & 4.00 & Triflumuron & 4.71 & FB2 & 4.00 & Procymidone & 4.18 & Teflubenzuron & 1.65 & Chlorothalonil & 0.97 & Sulphur & 1.29 \\
\hline Pyrene & 3.67 & PBB 153 & 3.97 & Folpet & 4.71 & Tartaric acid & 3.63 & Myclobutanil & 4.14 & Methomyl & 1.28 & Vanadium & 0.95 & Cyclopenta(c,d)pyrene & 1.14 \\
\hline Indeno[1,2,3-cd]pyrene & 3.67 & Organic arsenic & 3.97 & Methomyl & 4.54 & DON15 & 3.33 & Cyprodinyl & 3.81 & Metalaxyl & 1.21 & Cyproconazole & 0.80 & Cyproconazole & 1.11 \\
\hline OCDF & 3.60 & PFOS & 3.94 & Teflubenzuron & 4.39 & Hexachlorobenzene & 3.07 & Iprodione & 3.26 & Cyprodinyl & 1.02 & Inorganic arsenic & 0.74 & Chlorothalonil & 1.08 \\
\hline HCDF 234678 & 3.55 & PCB 169 & 3.92 & Chlorpyrifos-ethyl & 4.05 & Lindane & 2.81 & Lambda-cyhalothrin & 3.06 & PFHXA & 0.90 & PFOS & 0.67 & Diethofencarb & 1.08 \\
\hline HCDF 1234789 & 3.55 & TCDF 2378 & 3.80 & Fludioxonil & 3.92 & Phenylphenol & 2.77 & Metalaxyl & 2.88 & PFBS & 0.90 & Dibenzo[a,h]pyrene & 0.62 & Indeno[1,2,3-cd]pyrene & 0.89 \\
\hline Aluminium & 3.51 & PBB 52 & 3.57 & Boscalid & 3.88 & 5-methylchrysene & 2.70 & Boscalid & 2.46 & PFHXS & 0.90 & Bifenthrin & 0.42 & Pirimiphos-methyl & 0.87 \\
\hline HCDF 123478 & 3.51 & PCB 77 & 3.52 & Bifenthrin & 2.91 & Enterolactone & 2.50 & Chlorpyrifos-ethyl & 1.15 & PFHpA & 0.89 & Gallium & 0.41 & Flutriafol & 0.71 \\
\hline PFOA & 3.51 & PBB 101 & 3.48 & Patulin & 2.52 & Dibenzo[a,l]pyrene & 2.45 & Pyrimethanil & 1.06 & Cyproconazole & 0.84 & PFTeDA & 0.40 & Iprodione & 0.65 \\
\hline HCDF 1234678 & 3.48 & PCB 105 & 3.47 & Pyrimethanil & 1.95 & Equol & 2.42 & Tetradifon & 0.99 & Sulphur & 0.83 & PFDoA & 0.40 & Ethion & 0.63 \\
\hline Cyclopenta(c,d)pyrene & 3.46 & Methyl mercury & 3.38 & Cyprodinyl & 1.74 & Benzo[k]fluoranthene & 2.25 & Pyriproxyfen & 0.99 & Pyriproxyfen & 0.82 & Inorganic mercury & 0.40 & Sulphites & 0.61 \\
\hline Benzo[a]pyrene & 3.41 & PFUnA & 3.25 & Imazalil & 1.72 & Tin & 2.18 & Diethofencarb & 0.91 & Diethofencarb & 0.79 & Patulin & 0.39 & Bifenthrin & 0.51 \\
\hline Fluoranthene & 3.40 & PFTrDA & 3.23 & Iprodione & 1.24 & Formononetin & 1.82 & Chlorothalonil & 0.91 & Chlorothalonil & 0.79 & Antimony & 0.37 & HT2 & 0.47 \\
\hline \multicolumn{2}{|c|}{ Total contribution $=44 \%$} & \multicolumn{2}{|c|}{ Total contribution $=\mathbf{8 7 \%}$} & \multicolumn{2}{|c|}{ Total contribution $=95 \%$} & \multicolumn{2}{|c|}{ Total contribution $=\mathbf{7 2 . 7 \%}$} & \multicolumn{2}{|c|}{ Total contribution $=95 \%$} & \multicolumn{2}{|c|}{ Total contribution $=87.8 \%$} & \multicolumn{2}{|c|}{ Total contribution $=93.6 \%$} & \multicolumn{2}{|c|}{ Total contribution $=94.4 \%$} \\
\hline \multicolumn{2}{|c|}{79 substances with $\%$ Subst $>0$} & \multicolumn{2}{|c|}{42 substances with $\%$ Subst $>0$} & \multicolumn{2}{|c|}{42 substances with $\%$ Subst $>0$} & \multicolumn{2}{|c|}{71 substances with $\%$ Subst $>0$} & \multicolumn{2}{|c|}{47 substances with \%Subst >0 } & \multicolumn{2}{|c|}{78 substances with \%Subst $>0$} & \multicolumn{2}{|c|}{60 substances with $\%$ Subst $>0$} & \multicolumn{2}{|c|}{54 substances with \%Subst >0 } \\
\hline
\end{tabular}




\begin{tabular}{|c|c|c|c|c|c|c|c|c|c|c|c|c|c|c|c|}
\hline \multicolumn{16}{|c|}{ EDEN mixtures during pregnancy } \\
\hline TE-F-PAH (39.1\%) & \% Subst & Mixt-2 (19.5\%) & \% Subst & Pest-1 (7.8\%) & \% Subst & Pest-3 (4.2\%) & \% Subst & $\begin{array}{c}\text { PCB-BFR-Aso-MeHg } \\
(5.1 \%)\end{array}$ & \% Subst & Pest-2 (5.5\%) & \% Subst & Myco-Pest-PAH (7.2\%) & \% Subst & | PFAA-Ge-Li (11.5\%) & \% Subst \\
\hline Inorganic arsenic & 5.70 & PCB 126 & 4.74 & Phosalone & 6.23 & Myclobutanil & 6.26 & PFTrDA & 6.24 & Acrinathrin & 7.04 & Alpha-zearalanol & 12.2 & PFHXS & 15.5 \\
\hline Chromium VI & 5.56 & PCB 118 & 4.65 & Diphenylamine & 6.21 & Triadimenol & 6.09 & PFUnA & 6.15 & Endosulfan & 6.85 & Alpha-zearalanol & 12.2 & PFBS & 15.5 \\
\hline Vanadium & 5.37 & PCB 52 & 4.55 & Azinphos-methyl & 6.21 & Cyfluthrin & 6.07 & Methyl mercury & 5.97 & Bupirimate & 6.84 & DAS & 12.2 & PFHXA & 14.7 \\
\hline Germanium & 5.29 & PCB 114 & 4.43 & Tebufenozide & 6.21 & Tetraconazole & 6.07 & PBB 101 & 5.83 & Dichlorvos & 6.84 & DON3 & 12.2 & PFHpA & 14.3 \\
\hline Strontium & 4.94 & PCB 77 & 4.43 & Diflubenzuron & 6.21 & Spiroxamine & 6.07 & PBB 52 & 5.36 & Kresoxim-methyl & 6.65 & FusX & 12.2 & PFOA & 7.50 \\
\hline Chromium III & 4.34 & PCB 105 & 4.38 & Captan & 6.21 & Quinoxyfen & 6.07 & PBB 153 & 4.54 & Azoxystrobin & 6.47 & ОТВ & 4.61 & Strontium & 6.00 \\
\hline Tellurium & 4.29 & HBCD alpha & 4.32 & Triflumuron & 6.21 & Etofenprox & 6.07 & PBDE 100 & 4.42 & Penconazole & 5.15 & OTA & 4.23 & Germanium & 4.52 \\
\hline Lead & 4.23 & Chrysene & 4.30 & Folpet & 6.21 & Trifloxystrobin & 6.07 & PFOS & 4.41 & Tebuconazole & 4.87 & Chlorpyrifos-methyl & 3.93 & Lithium & 3.01 \\
\hline Cobalt & 3.92 & Benzo[a]anthracene & 4.28 & Pirimicarb & 6.21 & Tebufenpyrad & 6.07 & PBDE 154 & 4.23 & Dimethoate & 4.86 & Benzo[g,h,i]perylene & 3.58 & Formononetin & 2.56 \\
\hline HCDF 1234789 & 3.84 & Benzo[j]fluoranthene & 4.26 & Ethoxyquin & 6.19 & Fenhexamid & 6.03 & PBDE 28 & 4.17 & Fenbuconazole & 4.83 & HT-2 toxin & 2.83 & Equol & 2.38 \\
\hline HCDF 234678 & 3.78 & Benzo[b]fluoranthene & 4.07 & Propargite & 5.89 & Pyrimethanil & 5.15 & PCB 28 & 4.14 & Procymidone & 4.50 & Benzo[a]pyrene & 2.82 & Enterolactone & 2.29 \\
\hline HCDF 1234678 & 3.75 & PCB 169 & 4.01 & Thiabendazole & 5.49 & Bifenthrin & 4.22 & PCB 101 & 3.79 & Mepanipyrim & 4.38 & \begin{tabular}{|l} 
Pirimiphos-methyl \\
\end{tabular} & 2.81 & Matairesinol & 2.27 \\
\hline OCDF & 3.75 & Inorganic mercury & 3.99 & Carbendazim & 4.80 & Teflubenzuron & 4.21 & PBDE 153 & 3.61 & Boscalid & 4.02 & Cyclopenta(c,d)pyrene & 2.43 & Glycitein & 1.74 \\
\hline HCDF 123478 & 3.73 & TCDF 2378 & 3.97 & Phosmet & 4.29 & Methomyl & 3.95 & PCB 123 & 3.58 & Lambda-cyhalothrin & 3.96 & Indeno[1,2,3-cd]pyrene & 2.22 & Inorganic arsenic & 1.22 \\
\hline PFOA & 3.67 & Benzo[k]fluoranthene & 3.95 & Methomyl & 3.73 & Mepanipyrim & 3.92 & PBDE 99 & 3.52 & Cyprodinyl & 3.31 & DON & 1.68 & Vanadium & 1.05 \\
\hline Pyrene & 3.65 & Benzo[c]fluorene & 3.83 & Teflubenzuron & 3.43 & Cyprodinyl & 3.40 & Organic arsenic & 3.46 & Iprodione & 3.30 & Zearalenone & 1.07 & 5-methylchrysene & 0.98 \\
\hline HCDF 123678 & 3.51 & PCB 157 & 3.76 & Chlorpyrifos-ethyl & 2.63 & Penconazole & 3.08 & PCB 189 & 3.42 & Metalaxyl & 2.68 & Pyrene & 1.05 & DON15 & 0.74 \\
\hline Barium & 3.48 & Bisphenol A & 3.74 & Patulin & 1.79 & Metalaxyl & 1.99 & PCB 81 & 3.21 & Chlorpyrifos-ethyl & 2.62 & Daidzein & 0.87 & Chromium VI & 0.57 \\
\hline Aluminium & 3.40 & PCB 138 & 3.70 & Boscalid & 1.50 & Boscalid & 1.80 & PCB 180 & 3.19 & Fludioxonyl & 1.98 & Genistein & 0.85 & FB2 & 0.55 \\
\hline Nickel & 3.36 & PCB 156 & 3.64 & Fludioxonil & 1.46 & Chlorpyrifos-methyl & 1.73 & PCDF 12378 & 3.13 & Nickel & 1.84 & Gallium & 0.69 & Inorganic mercury & 0.55 \\
\hline Cadmium & 3.34 & РСB 153 & 3.54 & Ethion & 0.77 & \begin{tabular}{|l|} 
Chlorpyrifos-ethyl \\
\end{tabular} & 1.62 & PCB 167 & 2.98 & Silver & 1.84 & Cyproconazole & 0.68 & Tellurium & 0.52 \\
\hline Benzo[g,h,i]perylene & 3.34 & Dibenzo[a,e]pyrene & 3.41 & Imazalil & 0.64 & Iprodione & 1.40 & PBDE 47 & 2.94 & Phosmet & 1.80 & PFBS & 0.68 & Malathion & 0.43 \\
\hline HCDD 123678 & 3.26 & PCB 167 & 3.39 & Bifenthrin & 0.59 & Azoxystrobin & 1.09 & PCB 153 & 2.70 & Fenhexamid & 1.41 & PFHXS & 0.68 & Phenylphenol & 0.40 \\
\hline HCDD 1234678 & 3.26 & PBDE 47 & 3.33 & PFNA & 0.54 & Lambda-cyhalothrin & 0.91 & PCB 156 & 2.53 & Sulphites & 1.00 & DON15 & 0.65 & Carbaryl & 0.40 \\
\hline Fluoranthene & 3.24 & $T-2$ toxin & 3.31 & Daidzein & 0.32 & Silver & 0.63 & Inorganic mercury & 2.47 & Chlorothalonil & 0.97 & Sulphur & 0.64 & Resveratrol & 0.37 \\
\hline \multicolumn{2}{|c|}{ Total contribution $=\mathbf{5 1 . 8 \%}$} & \multicolumn{2}{|c|}{ Total contribution $=48.4 \%$} & \multicolumn{2}{|c|}{ Total contribution $=96.5 \%$} & \multicolumn{2}{|c|}{ Total contribution $=94.2 \%$} & \multicolumn{2}{|c|}{ Total contribution $=75.9 \%$} & \multicolumn{2}{|c|}{ Total contribution $=\mathbf{8 8 . 9 \%}$} & \multicolumn{2}{|c|}{ Total contribution $=91.6 \%$} & \multicolumn{2}{|c|}{ Total contribution $=93 \%$} \\
\hline \multicolumn{2}{|c|}{64 substances with \%Subst >0 } & \multicolumn{2}{|c|}{94 substances with $\%$ Subst $>0$} & \multicolumn{2}{|c|}{53 substances with $\%$ Subst $>0$} & \multicolumn{2}{|c|}{49 substances with \%Subst >0 } & \multicolumn{2}{|c|}{71 substances with $\%$ Subst $>0$} & \multicolumn{2}{|c|}{61 substances with $\%$ Subst $>0$} & \multicolumn{2}{|c|}{56 substances with \%Subst >0 } & \multicolumn{2}{|c|}{58 substances with \%Subst $>0$} \\
\hline
\end{tabular}




\begin{tabular}{|c|c|c|c|c|c|c|c|c|c|c|c|c|c|c|c|}
\hline \multicolumn{16}{|c|}{ ELFE mixtures during pregnancy } \\
\hline TE-F-PAH (31.4\%) & \% Subst & \begin{tabular}{|c|} 
PCB-BFR-Aso-MeHg \\
$(10.7 \%)$
\end{tabular} & \% Subst & Pest-1 (12.9\%) & \% Subst & Pest-3 (6.9\%) & \% Subst & PFAA-Li-Ge (16.0\%) & \% Subst & Pest-2 (7.5\%) & \% Subst & Mixt-3 (4.4\%) & \% Subst & Mixt-4 (10.2\%) & \% Subst \\
\hline Chromium VI & 4.92 & PCB 123 & 4.61 & Propargite & 6.27 & Myclobutanil & 5.71 & PFHXA & 13.9 & Bupirimate & 8.32 & PFTeDA & 7.57 & Chlorothalonil & 11.2 \\
\hline Chromium III & 4.66 & PBDE 47 & 4.46 & Thiabendazole & 6.17 & Fenhexamid & 5.64 & PFHpA & 13.9 & \begin{tabular}{|l} 
Dichlorvos \\
\end{tabular} & 8.28 & Benzo[k]fluoranthene & 7.55 & Diethofencarb & 11.2 \\
\hline Lead & 4.62 & PBDE 100 & 4.42 & Pirimicarb & 6.12 & Triadimenol & 5.48 & PFHxS & 13.8 & Endosulfan & 8.25 & Benzo[b]fluoranthene & 7.50 & Carbofuran & 11.2 \\
\hline Cobalt & 4.62 & PCB 52 & 4.42 & Phosalone & 6.10 & Etofenprox & 5.42 & PFBS & 13.8 & Acrinathrin & 8.24 & Benzo[j]fluoranthene & 7.31 & Tetradifon & 11.2 \\
\hline Barium & 4.43 & PCB 101 & 4.40 & Diphenylamine & 6.09 & Trifloxystrobin & 5.42 & PFOA & 10.4 & Kresoxim-methyl & 8.05 & Dibenzo[a,i]pyrene & 7.12 & Pyriproxyfen & 11.1 \\
\hline Tellurium & 4.43 & PBDE 154 & 4.40 & Diflubenzuron & 6.09 & Quinoxyfen & 5.42 & Carbendazim & 5.42 & Azoxystrobin & 7.42 & PFDoA & 6.34 & Chlorthal-dimethyl & 9.11 \\
\hline Vanadium & 4.38 & PCB 77 & 4.36 & Folpet & 6.09 & Spiroxamine & 5.42 & Chromium VI & 2.87 & \begin{tabular}{|l} 
Penconazole \\
\end{tabular} & 5.47 & Chrysene & 6.01 & Procymidone & 6.93 \\
\hline Nickel & 4.36 & PCB 153 & 4.30 & Azinphos-methyl & 6.09 & Cyfluthrin & 5.42 & FB1 & 2.42 & Boscalid & 4.91 & Benzo[a]anthracene & 4.94 & Pyrimethanil & 4.71 \\
\hline Inorganic arsenic & 4.15 & PBB 153 & 4.23 & Captan & 6.09 & Tetraconazole & 5.42 & OTA & 2.11 & Tebuconazole & 4.75 & Dibenzo[a,e]pyrene & 4.79 & Metalaxyl & 4.29 \\
\hline Antimony & 3.98 & PCB 105 & 4.23 & Ethoxyquin & 6.09 & Tebufenpyrad & 5.42 & FB2 & 2.04 & Dimethoate & 4.67 & Benzo[a]pyrene & 4.58 & Iprodione & 3.35 \\
\hline HCDF 123678 & 3.96 & PBDE 28 & 4.20 & Triflumuron & 6.09 & Methomyl & 4.88 & Lithium & 1.81 & Fenbuconazole & 4.65 & Silver & 4.16 & Bifenthrin & 2.54 \\
\hline Pyrene & 3.94 & PBB 52 & 4.15 & Tebufenozide & 6.09 & Teflubenzuron & 4.63 & Phenylphenol & 1.65 & Mepanipyrim & 4.33 & Indeno[1,2,3-cd]pyrene & 3.78 & Sulphur & 2.44 \\
\hline Aluminium & 3.89 & PCB 157 & 4.14 & Carbendazim & 5.86 & Pyrimethanil & 4.48 & Tin & 1.49 & Procymidone & 3.99 & Dibenzo[a,h]anthracene & 3.32 & Cyproconazole & 2.01 \\
\hline HCDF 123478 & 3.83 & TCDF 2378 & 4.07 & Phosmet & 3.54 & Cyprodinyl & 4.22 & Pyriproxyfen & 1.45 & Phosmet & 3.00 & HBCD beta & 2.74 & Tin & 1.33 \\
\hline HCDF 1234789 & 3.83 & PCB 167 & 4.02 & Patulin & 2.96 & Bifenthrin & 4.11 & PFOS & 1.40 & Chlorpyrifos-ethyl & 2.74 & PFDA & 2.74 & Cyprodinyl & 1.32 \\
\hline OCDF & 3.77 & PBB 101 & 3.98 & Fludioxonil & 2.45 & Mepanipyrim & 3.99 & Germanium & 1.30 & Metalaxyl & 2.66 & Dibenzo[a,l]pyrene & 2.61 & Lambda-cyhalothrin & 1.15 \\
\hline Lithium & 3.70 & PCB 114 & 3.85 & Chlorpyrifos-ethyl & 2.21 & Metalaxyl & 3.80 & Tetradifon & 1.18 & Lambda-cyhalothrin & 2.48 & PFOS & 2.45 & Fludioxonil & 1.10 \\
\hline HCDF 123789 & 3.68 & PCDF 12378 & 3.74 & Teflubenzuron & 1.75 & Penconazole & 3.23 & DON15 & 1.17 & Cyprodinyl & 2.11 & Benzo[c]fluorene & 2.30 & Secoisolariciresino & 0.71 \\
\hline HCDD 123678 & 3.64 & PCB 138 & 3.73 & Boscalid & 1.66 & Boscalid & 2.55 & Lindane & 1.15 & Fludioxonyl & 1.61 & Organic arsenic & 2.11 & Flutriafol & 0.68 \\
\hline HCDD 123789 & 3.62 & РCB 189 & 3.65 & Methomyl & 1.26 & | Iprodione & 2.03 & Sulphur & 1.15 & Iprodione & 1.23 & PFNA & 2.00 & Imidacloprid & 0.68 \\
\hline HCDF 1234678 & 3.60 & Organic arsenic & 3.51 & Bifenthrin & 1.14 & Chlorpyrifos-ethyl & 1.96 & Gallium & 1.14 & Fenhexamid & 0.67 & Benzo[g,h,i]perylene & 1.98 & Boscalid & 0.49 \\
\hline HCDF 234678 & 3.58 & Methyl mercury & 3.43 & Pyrimethanil & 1.06 & Lambda-cyhalothrin & 1.43 & Equol & 1.13 & Sulphites & 0.62 & Cadmium & 1.86 & Tebuconazole & 0.40 \\
\hline HCDD 123478 & 3.51 & PCB 118 & 3.39 & Phenylphenol & 0.96 & Procymidone & 1.37 & Carbofuran & 1.12 & Nickel & 0.62 & Fluoranthene & 1.76 & Lithium & 0.37 \\
\hline Cadmium & 3.51 & PCB 180 & 3.21 & Lambda-cyhalothrin & 0.88 & Azoxystrobin & 1.28 & Diethofencarb & 1.12 & Chlorthal-dimethyl & 0.49 & HBCD gamma & 1.26 & Chlorfenvinphos & 0.33 \\
\hline Zearalenone & 3.40 & PCB 156 & 3.10 & Cyprodinyl & 0.86 & Chlorpyrifos-methyl & 1.27 & Chlorothalonil & 1.12 & Pyriproxyfen & 0.43 & OCDD & 1.22 & Lindane & 0.28 \\
\hline \multicolumn{2}{|c|}{ Total contribution = $47 \%$} & \multicolumn{2}{|c|}{ Total contribution $=68 \%$} & \multicolumn{2}{|c|}{ Total contribution $=93.5 \%$} & \multicolumn{2}{|c|}{ Total contribution $=\mathbf{9 2 . 1 \%}$} & \multicolumn{2}{|c|}{ Total contribution $=82.8 \%$} & \multicolumn{2}{|c|}{ Total contribution = 95\% } & \multicolumn{2}{|c|}{ Total contribution $=85.2 \%$} & \multicolumn{2}{|c|}{ Total contribution $=97.2 \%$} \\
\hline \multicolumn{2}{|c|}{85 substances with $\%$ Subst $>0$} & \multicolumn{2}{|c|}{80 substances with $\%$ Subst $>0$} & \multicolumn{2}{|c|}{52 substances with $\%$ Subst >0 } & \multicolumn{2}{|c|}{58 substances with \%Subst >0 } & \multicolumn{2}{|c|}{72 substances with $\%$ Subst $>0$} & \multicolumn{2}{|c|}{58 substances with $\%$ Subst $>0$} & \multicolumn{2}{|c|}{62 substances with $\%$ Subst $>0$} & \multicolumn{2}{|c|}{50 substances with $\%$ Subst >0 } \\
\hline
\end{tabular}




\subsection{ELFE exposure profiles}

The clustering method applied to matrix $H$ from the factorisation of the ELFE exposure matrix led to division of the 15,226 subjects in the overall study population into six clusters. A cluster is based on women with similar co-exposure profiles. For each cluster, co-exposure profiles were linked to the identified mixtures of the ELFE cohort presented in Table 3. Within each cluster, mixtures covering at least $65 \%$ of the total exposure were described. The exposure (mean, P95 and P99) of the cluster and of the whole population was assessed for each mixture compound (Table 4). The average age, BMI before pregnancy and weight gain during pregnancy are also presented (Table 4). The whole study population of 15,226 pregnant women in France had a mean age of 30.7 years $\left[P_{2.5} ; P_{97.5}\right]=[21.1 ; 40.7]$, a mean BMI before pregnancy of $23.5 \mathrm{~kg} / \mathrm{m}^{2}\left[P_{2.5} ; P_{97.5}\right]=$ $[17.6 ; 36.3]$, and a mean weight gain during pregnancy of $13.1 \mathrm{~kg}\left[P_{2.5} ; P_{97.5}\right]=[2.0 ; 25.0]$.

Cluster 1 contains 5,090 women (33.4\%) with a mean age of 30.4 years and a mean weight gain during pregnancy of $13.0 \mathrm{~kg}$. This cluster is characterised at $36.6 \%$ by "PFAA-Ge-Li" mixture and TEF-PAH" mixture "at 31\%. Significantly higher exposures were observed for PFAAs and carbendazim from mixture "PFAA-Ge-Li" for individuals in this cluster compared to the whole ELFE population.

Members of cluster 2 , which represents nearly $10 \%$ (1,520 women) of the overall study population, were significantly older (mean age of 32.0 years) and had a mean BMI $\left(22.9 \mathrm{~kg} / \mathrm{m}^{2}\right)$ significantly lower than the whole ELFE population. This cluster is characterised by the "Pest-1" mixture (40.5\%) and "TE-F-PAH" mixture (24.8\%). Individuals in this cluster were significantly more exposed to substances from "Pest-1" mixture compared to the whole ELFE population.

Cluster 3 is composed of 5,183 women (34\%) with a mean age of 30.1 and a mean BMI of $23.6 \mathrm{~kg} / \mathrm{m}^{2}$. This cluster is characterised by "TE-F-PAH" mixture (49.5\%), PCB-BFR-Aso-MeHg" mixture "(11.4\%) and "Pest-1" mixture (9.9\%). This cluster has the specificity of having no significantly higher exposure compared to the whole population, irrespective of the substance.

Cluster 4 , composed of 1,039 women $(6.8 \%)$ with a mean age of 31.0 years was characterised by "TE-F-PAH" mixture (37.8\%), "PCB-BFR-Aso-MeHg mixture" (24\%) and "Mixt-3" mixture (9.7\%). 
Individuals in this cluster were significantly more exposed to all selected substances in these mixtures than the overall study population.

Cluster 5 includes $7 \%$ of the whole population. Members were significantly older than the overall study population (31.4 years old on average). Individuals in this cluster were mostly characterised by "Pest-3" mixture (21.4\%), "Te-F-PAH" mixture (19.9\%) and "Pest-2" mixture (18.6\%). Women in this cluster were also significantly more exposed to all substances in these mixtures than the whole ELFE population.

Cluster 6 is composed of women significantly older (mean age of 31.8 years) and with a lower mean BMI $\left(23.1 \mathrm{~kg} / \mathrm{m}^{2}\right)$ than the whole ELFE population. This cluster was associated to "Mixt-4" mixture (38.2\%), "TE-F-PAH" mixture (24.4\%) and "PFAA-Ge-Li" mixture (13.3\%). Members of this cluster were significantly more exposed to substances from "Mixt-4" mixture compared to the whole ELFE population. 


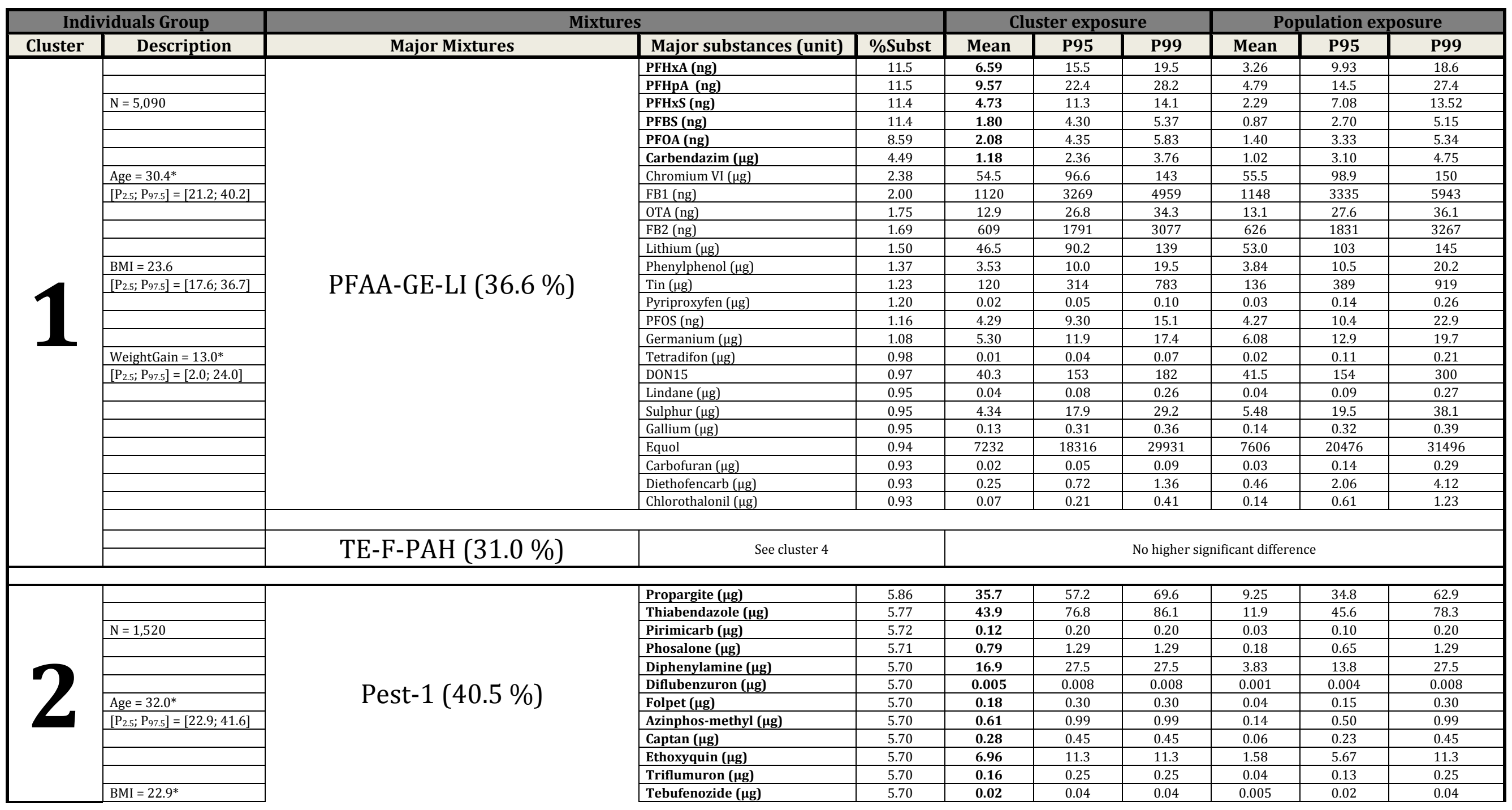




\begin{tabular}{|c|c|c|c|c|c|c|c|c|c|c|}
\hline \multicolumn{2}{|c|}{ Individuals Group } & \multicolumn{3}{|c|}{ Mixtures } & \multicolumn{3}{|c|}{ Cluster exposure } & \multicolumn{3}{|c|}{ Population exposure } \\
\hline Cluster & Description & \multirow[t]{14}{*}{ Major Mixtures } & Major substances (unit) & \multirow{2}{*}{$\frac{\text { \%Subst }}{5.48}$} & \multirow{2}{*}{$\begin{array}{c}\text { Mean } \\
2.51\end{array}$} & \multirow{2}{*}{$\begin{array}{c}\text { P95 } \\
4.25 \\
\end{array}$} & \multirow{2}{*}{\begin{tabular}{|l|} 
P99 \\
4.74 \\
\end{tabular}} & \multirow{2}{*}{$\begin{array}{c}\text { Mean } \\
1.02\end{array}$} & \multirow{2}{*}{$\begin{array}{c}\text { P95 } \\
3.10 \\
\end{array}$} & \multirow{2}{*}{$\begin{array}{c}\text { P99 } \\
4.75\end{array}$} \\
\hline & {$\left[\mathrm{P}_{2.5} ; \mathrm{P}_{97.5}\right]=[17.6 ; 35.2]$} & & \multirow{2}{*}{\begin{tabular}{l|l} 
Carbendazim $(\mu \mathrm{g})$ \\
Phosmet $(\mu \mathrm{g})$
\end{tabular}} & & & & & & & \\
\hline & \multirow{5}{*}{\begin{tabular}{|l|} 
WeightGain $=13.1$ \\
{$\left[\mathrm{P}_{2.5} ; \mathrm{P}_{97.5}\right]=[17.6 ; 35.2]$} \\
\end{tabular}} & & & 3.31 & 0.67 & 1.20 & 2.39 & 0.28 & 1.20 & 1.96 \\
\hline & & & \begin{tabular}{|l|} 
Patulin (ng) \\
\end{tabular} & 2.77 & 72.9 & 148 & 205 & 40.9 & 111 & 215 \\
\hline & & & \begin{tabular}{|l} 
Fludioxonil $(\mu \mathrm{g})$ \\
\end{tabular} & 2.29 & 6.34 & 16.0 & 23.0 & 3.58 & 12.2 & 22.4 \\
\hline & & & Chlorpyrifos-ethyl $(\mu \mathrm{g})$ & 2.07 & 1.82 & 4.39 & 7.95 & 1.13 & 4.92 & 8.94 \\
\hline & & & Teflubenzuron $(\mu \mathrm{g})$ & 1.64 & 0.16 & 0.28 & 0.36 & 0.10 & 0.36 & 1.26 \\
\hline & & & \begin{tabular}{|l|} 
Boscalid $(\mu \mathrm{g})$ \\
\end{tabular} & 1.55 & 1.64 & 3.32 & 4.80 & 1.38 & 4.73 & 10.9 \\
\hline & & & \begin{tabular}{|l} 
Methomyl $(\mu \mathrm{g})$ \\
\end{tabular} & 1.18 & 0.007 & 0.01 & 0.02 & 0.005 & 0.02 & 0.07 \\
\hline & & & \begin{tabular}{|l|} 
Bifenthrin $(\mu \mathrm{g})$ \\
\end{tabular} & 1.07 & 0.20 & 0.57 & 0.89 & 0.18 & 0.65 & 1.23 \\
\hline & & & \begin{tabular}{|l} 
Pyrimethanil $(\mu \mathrm{g})$ \\
\end{tabular} & 0.99 & 1.91 & 5.34 & 7.22 & 1.80 & 6.66 & 13.7 \\
\hline & & & \begin{tabular}{|l} 
Phenylphenol ( $\mu \mathrm{g})$ \\
\end{tabular} & 0.90 & 4.16 & 10.4 & 16.8 & 3.84 & 10.5 & 20.2 \\
\hline & & & Lambda-cyhalothrin $(\mu \mathrm{g})$ & 0.82 & 0.46 & 1.41 & 2.27 & 0.37 & 1.32 & 2.15 \\
\hline & & & \begin{tabular}{|l|} 
Cyprodinyl $(\mu \mathrm{g})$ \\
\end{tabular} & 0.80 & 2.03 & 5.63 & 8.41 & 2.16 & 7.68 & 15.2 \\
\hline & & TE-F-PAH (24.8\%) & See cluster 4 & & & & Jo higher & îcant differ & & \\
\hline & & & Chromium VI $(\mu \mathrm{g})$ & 2.31 & 47.5 & 81.3 & 102 & 55.5 & 98.9 & 150 \\
\hline & & & \begin{tabular}{|l} 
Chromium III $(\mu \mathrm{g})$ \\
\end{tabular} & 2.19 & 291 & 506 & 685 & 338 & 626 & 1041 \\
\hline & $\mathrm{N}=5,183$ & & \begin{tabular}{|l|} 
Lead $(\mu \mathrm{g})$ \\
\end{tabular} & 2.17 & 13.0 & 21.5 & 29.1 & 15.7 & 27.9 & 44.7 \\
\hline & & & \begin{tabular}{|l|} 
Cobalt $(\mu \mathrm{g})$ \\
\end{tabular} & 2.17 & 13.6 & 23.2 & 29.7 & 15.8 & 28.8 & 46.2 \\
\hline & & & \begin{tabular}{|l} 
Barium $(\mu \mathrm{g})$ \\
\end{tabular} & 2.08 & 562 & 953 & 1241 & 653 & 1203 & 1975 \\
\hline & & & Tellurium $(\mu \mathrm{g})$ & 2.08 & 2.28 & 3.88 & 4.98 & 2.36 & 4.25 & 6.19 \\
\hline & & & Vanadium $(\mu \mathrm{g})$ & 2.06 & 73.0 & 131 & 176 & 77.9 & 147 & 224 \\
\hline & Age $=30.1^{*}$ & & \begin{tabular}{|l|} 
Nickel $(\mu \mathrm{g})$ \\
\end{tabular} & 2.05 & 154 & 265 & 348 & 194 & 361 & 601 \\
\hline & {$\left[\mathrm{P}_{2.5} ; \mathrm{P}_{97.5}\right]=[20.7 ; 40.3]$} & & \begin{tabular}{|l} 
Inorganic arsenic $(\mu \mathrm{g})$ \\
\end{tabular} & 1.95 & 30.6 & 54.9 & 74.1 & 31.9 & 60.8 & 95.8 \\
\hline & & & Antimony $(\mu \mathrm{g})$ & 1.87 & 1.92 & 3.61 & 5.23 & 2.13 & 4.20 & 6.94 \\
\hline & & & HCDF $123678(\mathrm{pg})$ & 1.86 & 4.29 & 7.76 & 9.95 & 5.16 & 9.74 & 15.9 \\
\hline & & & \begin{tabular}{|l} 
Pyrene (ng) \\
\end{tabular} & 1.85 & 414 & 764 & 1003 & 482 & 919 & 1469 \\
\hline & & TE-F-PAH (34 \%) & \begin{tabular}{|l} 
Aluminium $(\mu \mathrm{g})$ \\
\end{tabular} & 1.83 & 3012 & 5655 & 8073 & 3591 & 7017 & 12051 \\
\hline & BMI $=23.6^{*}$ & & \begin{tabular}{|l|} 
HCDF $123478(\mathrm{pg})$ \\
\end{tabular} & 1.80 & 7.32 & 13.0 & 16.9 & 8.79 & 16.4 & 28.2 \\
\hline & {$\left[\mathrm{P}_{2.5} ; \mathrm{P}_{97.5}\right]=[17.6 ; 36.3]$} & & HCDF $1234789(\mathrm{pg})$ & 1.80 & 5.23 & 9.33 & 11.7 & 6.22 & 11.6 & 19.6 \\
\hline & & & \begin{tabular}{|l|} 
OCDF (pg) \\
\end{tabular} & 1.77 & 25.6 & 45.7 & 57.3 & 30.5 & 57.3 & 94.6 \\
\hline & & & \begin{tabular}{|l|} 
Lithium $(\mu \mathrm{g})$ \\
\end{tabular} & 1.74 & 48.8 & 91.3 & 116 & 53.0 & 103 & 145 \\
\hline & & & HCDF 123789 (pg) & 1.73 & 1.06 & 1.96 & 2.48 & 1.30 & 2.50 & 4.11 \\
\hline & & & HCDD $123678(\mathrm{pg})$ & 1.71 & 5.94 & 10.9 & 14.2 & 7.22 & 14.0 & 24.0 \\
\hline & WeightGain $=13.2$ & & HCDD 123789 (pg) & 1.70 & 2.07 & 3.87 & 4.97 & 2.50 & 4.87 & 7.81 \\
\hline & {$\left[\mathrm{P}_{2.5} ; \mathrm{P}_{97.5}\right]=[1.0 ; 25.0]$} & & HCDF $1234678(\mathrm{pg})$ & 1.69 & 9.31 & 16.8 & 21.9 & 11.2 & 21.1 & 37.3 \\
\hline & & & HCDF $234678(\mathrm{pg})$ & 1.68 & 2.02 & 3.56 & 4.42 & 2.46 & 4.57 & 7.91 \\
\hline & & & HCDD $123478(\mathrm{pg})$ & 1.65 & 1.58 & 2.94 & 3.86 & 1.90 & 3.74 & 6.35 \\
\hline & & & Cadmium $(\mu \mathrm{g})$ & 1.65 & 10.9 & 18.7 & 24.6 & 13.4 & 25.0 & 44.8 \\
\hline & & & Zearalenone (ng) & 1.60 & 438 & 838 & 1197 & 489 & 983 & 1619 \\
\hline & & & & & & & & & & \\
\hline & & & PCB $123(\mathrm{pg})$ & 3.14 & 604 & 1423 & 1990 & 960 & 2580 & 4575 \\
\hline & & & PBDE 47 (ng) & 3.04 & 8.31 & 20.3 & 29.1 & 13.6 & 37.4 & 68.9 \\
\hline & & PCB-BFR-Aso-MeHg (11.4\%) & PBDE $100(\mathrm{ng})$ & 3.01 & 1.68 & 4.34 & 6.30 & 2.82 & 8.07 & 14.7 \\
\hline & & & PCB $52(\mathrm{pg})$ & 3.01 & 9784 & 22288 & 31568 & 15425 & 39948 & 79671 \\
\hline & & & PCB $101(\mathrm{pg})$ & 3.00 & 14632 & 38057 & 56236 & 24856 & 71555 & 136572 \\
\hline
\end{tabular}




\begin{tabular}{|c|c|c|c|c|c|c|c|c|c|c|}
\hline \multicolumn{2}{|c|}{ Individuals Group } & \multicolumn{3}{|c|}{ Mixtures } & \multicolumn{3}{|c|}{ Cluster exposure } & \multicolumn{3}{|c|}{ Population exposure } \\
\hline Cluster & Description & Major Mixtures & Major substances (unit) & \%Subst & Mean & P95 & P99 & Mean & P95 & P99 \\
\hline & & & PBDE 154 (ng) & 3.00 & 0.88 & 2.28 & 3.31 & 1.48 & 4.21 & 7.76 \\
\hline & & & \begin{tabular}{|l|} 
PCB 77 (pg) \\
\end{tabular} & 2.97 & 247 & 543 & 736 & 385 & 962 & 1974 \\
\hline & & & \begin{tabular}{|l|} 
PCB $153(\mathrm{pg})$ \\
\end{tabular} & 2.93 & 56917 & 122694 & 158868 & 85576 & 208026 & 380119 \\
\hline & & & \begin{tabular}{|l|} 
PBB 153 (ng) \\
\end{tabular} & 2.88 & $\begin{array}{l}0.04 \\
\end{array}$ & 0.10 & 0.15 & 0.06 & 0.19 & $\begin{array}{l}0.38 \\
\end{array}$ \\
\hline & & & \begin{tabular}{|l|} 
PCB $105(\mathrm{pg})$ \\
\end{tabular} & 2.88 & 6488 & 13891 & 18032 & 9718 & 23474 & 43399 \\
\hline & & & PBDE 28 (ng) & 2.86 & 0.48 & 1.35 & 2.06 & 0.85 & 2.55 & 4.79 \\
\hline & & & \begin{tabular}{|l|} 
PBB 52 (ng) \\
\end{tabular} & 2.83 & 0.05 & 0.16 & 0.25 & 0.10 & 0.31 & 0.59 \\
\hline & & & PCB 157 (pg) & 2.82 & 664 & 1421 & 1810 & 992 & 2378 & 4334 \\
\hline & & & \begin{tabular}{|l|l|} 
TCDF $2378(\mathrm{pg})$ \\
\end{tabular} & 2.77 & 9.56 & 23.3 & 33.3 & 16.0 & 43.2 & 91.5 \\
\hline & & & \begin{tabular}{|l|} 
PCB $167(\mathrm{pg})$ \\
\end{tabular} & 2.74 & 1626 & 3444 & 4438 & 2409 & 5747 & 10425 \\
\hline & & & \begin{tabular}{|l|} 
PBB $101(\mathrm{ng})$ \\
\end{tabular} & 2.71 & 0.03 & 0.09 & 0.14 & 0.05 & 0.16 & 0.32 \\
\hline & & & \begin{tabular}{|l} 
PCB 114 (pg) \\
\end{tabular} & 2.62 & 502 & 1061 & 1350 & 741 & 1765 & 3282 \\
\hline & & & \begin{tabular}{|l} 
PCDF $12378(\mathrm{pg})$ \\
\end{tabular} & 2.55 & 1.96 & 3.95 & 5.18 & 2.91 & 6.77 & 13.9 \\
\hline & & & \begin{tabular}{|l|l} 
PCB $138(\mathrm{pg})$ \\
\end{tabular} & 2.54 & 40495 & 84856 & 106790 & 59247 & 139002 & 251234 \\
\hline & & & PCB 189 (pg) & 2.49 & 282 & 597 & 755 & 417 & 983 & $\begin{array}{ll}1828 \\
\end{array}$ \\
\hline & & & Organic arsenic $(\mu \mathrm{g})$ & 2.39 & 33.5 & 83.1 & 111 & 54.6 & 142 & 316 \\
\hline & & & \begin{tabular}{|l} 
Methyl mercury $(\mu \mathrm{g})$ \\
\end{tabular} & 2.34 & 1.05 & 3.41 & 4.70 & 1.82 & 5.45 & 9.99 \\
\hline & & & \begin{tabular}{|l|l|} 
PCB $118(\mathrm{pg})$ \\
\end{tabular} & 2.31 & 22533 & 45994 & 57252 & 32575 & 74781 & 137130 \\
\hline & & & \begin{tabular}{|l|} 
PCB $180(\mathrm{pg})$ \\
\end{tabular} & 2.19 & 20426 & 42775 & 53276 & 29689 & 69185 & 124568 \\
\hline & & & PCB $156(\mathrm{pg})$ & 2.11 & 2926 & 6038 & 7461 & 4225 & 9708 & 17959 \\
\hline & & & \begin{tabular}{|l|} 
Propargite $(\mu \mathrm{g})$ \\
\end{tabular} & 5.86 & 3.96 & 9.75 & 14.6 & 9.25 & 34.8 & 62.9 \\
\hline & & & Thiabendazole $(\mu \mathrm{g})$ & 5.77 & 5.80 & 15.4 & 21.3 & 11.9 & 45.6 & 78.3 \\
\hline & & & \begin{tabular}{|l|} 
Pirimicarb $(\mu \mathrm{g})$ \\
\end{tabular} & 5.72 & 0.01 & 0.03 & 0.03 & 0.03 & 0.10 & 0.20 \\
\hline & & & \begin{tabular}{|l} 
Phosalone $(\mu \mathrm{g})$ \\
\end{tabular} & 5.71 & 0.07 & 0.18 & 0.18 & 0.18 & 0.65 & 1.29 \\
\hline & & & Diphenylamine $(\mu \mathrm{g})$ & 5.70 & 1.54 & 3.86 & 3.86 & 3.83 & 13.8 & 27.5 \\
\hline & & & Diflubenzuron $(\mu \mathrm{g})$ & 5.70 & 0.001 & 0.001 & 0.001 & 0.001 & 0.004 & 0.008 \\
\hline & & & \begin{tabular}{|l} 
Folpet $(\mu \mathrm{g})$ \\
\end{tabular} & 5.70 & 0.02 & 0.04 & 0.04 & 0.04 & 0.15 & 0.30 \\
\hline & & & Azinphos-methyl ( $\mu \mathrm{g})$ & 5.70 & 0.06 & 0.14 & 0.14 & 0.14 & 0.50 & 0.99 \\
\hline & & & Captan $(\mu \mathrm{g})$ & 5.70 & 0.03 & 0.06 & 0.06 & 0.06 & 0.23 & 0.45 \\
\hline & & & \begin{tabular}{|l} 
Ethoxyquin $(\mu \mathrm{g})$ \\
\end{tabular} & 5.70 & 0.63 & 1.59 & 1.59 & 1.58 & 5.67 & 11.3 \\
\hline & & & \begin{tabular}{|l} 
Triflumuron $(\mu \mathrm{g})$ \\
\end{tabular} & 5.70 & 0.01 & 0.04 & 0.04 & 0.04 & 0.13 & 0.25 \\
\hline & & & \begin{tabular}{|l|} 
Tebufenozide $(\mu \mathrm{g})$ \\
\end{tabular} & 5.70 & 0.002 & 0.01 & 0.01 & 0.005 & 0.02 & 0.04 \\
\hline & & Pest-1 (9.9\%) & Carbendazim $(\mu \mathrm{g})$ & 5.48 & 0.28 & 0.69 & 0.85 & 1.02 & 3.10 & 4.75 \\
\hline & & & \begin{tabular}{|l} 
Phosmet $(\mu \mathrm{g})$ \\
\end{tabular} & 3.31 & 0.14 & 0.34 & 1.52 & 0.28 & 1.20 & 1.96 \\
\hline & & & \begin{tabular}{|l} 
Patulin (ng) \\
\end{tabular} & 2.77 & 33.0 & 105 & 210 & 40.9 & 111 & 215 \\
\hline & & & \begin{tabular}{|l|} 
Fludioxonil $(\mu \mathrm{g})$ \\
\end{tabular} & 2.29 & 1.93 & 5.11 & 10.3 & 3.58 & 12.2 & 22.4 \\
\hline & & & \begin{tabular}{|l} 
Chlorpyrifos-ethyl $(\mu \mathrm{g})$ \\
\end{tabular} & 2.07 & 0.59 & 1.55 & 6.27 & 1.13 & 4.92 & 8.94 \\
\hline & & & \begin{tabular}{|l} 
Teflubenzuron $(\mu \mathrm{g})$ \\
\end{tabular} & 1.64 & 0.04 & 0.18 & 0.19 & 0.10 & 0.36 & 1.26 \\
\hline & & & Boscalid $(\mu \mathrm{g})$ & 1.55 & 0.71 & 1.96 & 2.84 & 1.38 & 4.73 & 10.9 \\
\hline & & & \begin{tabular}{|l} 
Methomyl $(\mu \mathrm{g})$ \\
\end{tabular} & 1.18 & 0.002 & 0.01 & 0.01 & 0.005 & 0.02 & 0.07 \\
\hline & & & Bifenthrin $(\mu \mathrm{g})$ & 1.07 & 0.09 & 0.24 & 0.39 & 0.18 & 0.65 & 1.23 \\
\hline & & & \begin{tabular}{|l|} 
Pyrimethanil $(\mu \mathrm{g})$ \\
\end{tabular} & 0.99 & 0.78 & 2.17 & 2.81 & 1.80 & 6.66 & 13.7 \\
\hline & & & \begin{tabular}{|l|} 
Phenylphenol $(\mu \mathrm{g})$ \\
\end{tabular} & 0.90 & 3.46 & 9.96 & 19.4 & 3.84 & 10.5 & 20.2 \\
\hline & & & \begin{tabular}{|l} 
Lambda-cyhalothrin $(\mu \mathrm{g})$ \\
\end{tabular} & 0.82 & 0.22 & 0.70 & 1.28 & 0.37 & 1.32 & 2.15 \\
\hline & & & Cyprodinyl $(\mu \mathrm{g})$ & 0.80 & 1.16 & 3.29 & 4.97 & 2.16 & 7.68 & 15.2 \\
\hline & & & \begin{tabular}{|l|} 
Chromium VI ( $\mu \mathrm{g})$ \\
\end{tabular} & 2.31 & 69.4 & 129 & 166 & 55.5 & 98.9 & 150 \\
\hline & & & \begin{tabular}{|l|} 
Chromium III ( $\mu \mathrm{g})$ \\
\end{tabular} & 2.19 & 474 & 966 & 1356 & 338 & 626 & 1041 \\
\hline
\end{tabular}




\begin{tabular}{|c|c|c|c|c|c|c|c|c|c|c|}
\hline \multicolumn{2}{|c|}{ Individuals Group } & \multicolumn{3}{|c|}{ Mixtures } & \multicolumn{3}{|c|}{ Cluster exposure } & \multicolumn{3}{|c|}{ Population exposure } \\
\hline Cluster & Description & Major Mixtures & Major substances (unit) & \%Subst & Mean & P95 & P99 & Mean & P95 & P99 \\
\hline \multirow{48}{*}{2} & $\mathrm{~N}=1,039$ & \multirow{23}{*}{ TE-F-PAH (37.8 \%) } & \begin{tabular}{|l} 
Lead $(\mu \mathrm{g})$ \\
\end{tabular} & 2.17 & 20.7 & 37.7 & 48.8 & 15.7 & 27.9 & 44.7 \\
\hline & & & \begin{tabular}{|l} 
Cobalt $(\mu \mathrm{g})$ \\
\end{tabular} & 2.17 & 21.4 & 41.6 & 58.6 & 15.8 & 28.8 & 46.2 \\
\hline & & & Barium $(\mu \mathrm{g})$ & 2.08 & 858 & 1686 & 2310 & 653 & 1203 & 1975 \\
\hline & & & \begin{tabular}{|l|} 
Tellurium $(\mu \mathrm{g})$ \\
\end{tabular} & 2.08 & 3.12 & 5.59 & 7.30 & 2.36 & 4.25 & 6.19 \\
\hline & & & \begin{tabular}{|l} 
Vanadium $(\mu \mathrm{g})$ \\
\end{tabular} & 2.06 & 103 & 195 & 276 & 77.9 & 147 & 224 \\
\hline & Age $=31.0^{*}$ & & \begin{tabular}{|l|} 
Nickel $(\mu \mathrm{g})$ \\
\end{tabular} & 2.05 & 249 & 496 & 699 & 194 & 361 & 601 \\
\hline & {$\left[\mathrm{P}_{2.5} ; \mathrm{P}_{97.5}\right]=[21.1 ; 41.1]$} & & \begin{tabular}{|l|} 
Inorganic arsenic $(\mu \mathrm{g})$ \\
\end{tabular} & 1.95 & 43.9 & 82.9 & 116 & 31.9 & 60.8 & 95.8 \\
\hline & & & \begin{tabular}{|l|} 
Antimony $(\mu \mathrm{g})$ \\
\end{tabular} & 1.87 & 2.90 & 6.08 & 8.37 & 2.13 & 4.20 & 6.94 \\
\hline & & & HCDF $123678(\mathrm{pg})$ & 1.86 & 8.17 & 15.6 & 23.5 & 5.16 & 9.74 & 15.9 \\
\hline & & & \begin{tabular}{|l|} 
Pyrene (ng) \\
\end{tabular} & 1.85 & 737 & 1453 & 2159 & 482 & 919 & 1469 \\
\hline & & & \begin{tabular}{|l} 
Aluminium $(\mu \mathrm{g})$ \\
\end{tabular} & 1.83 & 4993 & 10226 & 13818 & 3591 & 7017 & 12051 \\
\hline & BMI $=23.7$ & & HCDF 123478 (pg) & 1.80 & 14.2 & 28.2 & 41.0 & 8.79 & 16.4 & 28.2 \\
\hline & {$\left[\mathrm{P}_{2.5} ; \mathrm{P}_{97.5}\right]=[21.1 ; 41.1]$} & & HCDF $1234789(\mathrm{pg})$ & 1.80 & 9.99 & 18.9 & 28.0 & 6.22 & 11.6 & 19.6 \\
\hline & & & OCDF (pg) & 1.77 & 49.6 & 91.9 & 137 & 30.5 & 57.3 & 94.6 \\
\hline & & & Lithium ( $\mu \mathrm{g})$ & 1.74 & 67.2 & 126 & 153 & 53.0 & 103 & 145 \\
\hline & & & HCDF 123789 (pg) & 1.73 & 2.16 & 3.96 & 5.80 & 1.30 & 2.50 & 4.11 \\
\hline & & & \begin{tabular}{|l} 
HCDD $123678(\mathrm{pg})$ \\
\end{tabular} & 1.71 & 11.8 & 23.2 & 33.7 & 7.22 & 14.0 & 24.0 \\
\hline & WeightGain $=13.3$ & & \begin{tabular}{|l|} 
HCDD $123789(\mathrm{pg})$ \\
\end{tabular} & 1.70 & 3.96 & 7.61 & 11.5 & 2.50 & 4.87 & 7.81 \\
\hline & {$\left[\mathrm{P}_{2.5} ; \mathrm{P}_{97.5}\right]=[2.0 ; 26.4]$} & & HCDF $1234678(\mathrm{pg})$ & 1.69 & 18.3 & 36.8 & 56.7 & 11.2 & 21.1 & 37.3 \\
\hline & & & HCDF 234678 (pg) & 1.68 & 4.10 & 7.56 & 10.9 & 2.46 & 4.57 & 7.91 \\
\hline & & & HCDD 123478 (pg) & 1.65 & 3.03 & 6.10 & 9.07 & 1.90 & 3.74 & 6.35 \\
\hline & & & \begin{tabular}{|l|} 
Cadmium $(\mu \mathrm{g})$ \\
\end{tabular} & 1.65 & 19.8 & 41.0 & 59.7 & 13.4 & 25.0 & 44.8 \\
\hline & & & Zearalenone (ng) & 1.60 & 676 & 1495 & 2183 & 489 & 983 & 1619 \\
\hline & & \multirow{25}{*}{ PCB-BFR-Aso-MeHg (24 \%) } & РСB 123 (pg) & 3.14 & 2494 & 5559 & 8654 & 960 & 2580 & 4575 \\
\hline & & & \begin{tabular}{|l} 
PBD 120 (pg) \\
PBDE 4 (ng) \\
\end{tabular} & $\begin{array}{l}0.14 \\
3.04\end{array}$ & 36.2 & 85.0 & $\begin{array}{c}0054 \\
128\end{array}$ & 13.6 & 37.4 & 68.9 \\
\hline & & & \begin{tabular}{|l|} 
PBDE 100 (ng) \\
\end{tabular} & 3.01 & 7.77 & 18.4 & 28.6 & 2.82 & 8.07 & 14.7 \\
\hline & & & \begin{tabular}{|l|} 
PCB 52 (pg) \\
\end{tabular} & 3.01 & 40056 & 89801 & 131602 & 15425 & 39948 & 79671 \\
\hline & & & PCB 101 (pg) & 3.00 & 69910 & 168748 & 243983 & 24856 & 71555 & 136572 \\
\hline & & & \begin{tabular}{|l|} 
PBDE 154 (ng) \\
\end{tabular} & 3.00 & 4.06 & 9.57 & 14.93 & 1.48 & 4.21 & 7.76 \\
\hline & & & \begin{tabular}{|l|l} 
PCB 77 (pg) \\
\end{tabular} & 2.97 & 989 & 2129 & 3178 & 385 & 962 & 1974 \\
\hline & & & PCB 153 (pg) & 2.93 & 206682 & 453129 & 642501 & 85576 & 208026 & 380119 \\
\hline & & & PBB 153 (ng) & 2.88 & 0.18 & 0.43 & 0.63 & 0.06 & 0.19 & 0.38 \\
\hline & & & PCB 105 (pg) & 2.88 & 23300 & 50623 & 72770 & 9718 & 23474 & 43399 \\
\hline & & & \begin{tabular}{|l|} 
PBDE 28 (ng) \\
\end{tabular} & 2.86 & 2.45 & 6.15 & 9.13 & 0.85 & 2.55 & 4.79 \\
\hline & & & \begin{tabular}{|l|} 
PBB 52 (ng) \\
\end{tabular} & 2.83 & 0.30 & 0.75 & 1.15 & 0.10 & 0.31 & 0.59 \\
\hline & & & PCB 157 (pg) & 2.82 & 2369 & 5141 & 7359 & 992 & 2378 & 4334 \\
\hline & & & \begin{tabular}{|l} 
TCDF $2378(\mathrm{pg})$ \\
\end{tabular} & 2.77 & 44.5 & 99.7 & 153 & 16.0 & 43.2 & 91.5 \\
\hline & & & \begin{tabular}{|l|l|} 
PCB 167 (pg) \\
\end{tabular} & 2.74 & 5703 & 12236 & 17889 & 2409 & 5747 & 10425 \\
\hline & & & PBB 101 (ng) & 2.71 & 0.16 & 0.40 & 0.62 & 0.05 & 0.16 & 0.32 \\
\hline & & & PCB 114 (pg) & 2.62 & 1746 & 3763 & 5356 & 741 & 1765 & 3282 \\
\hline & & & \begin{tabular}{|l} 
PCDF $12378(\mathrm{pg})$ \\
\end{tabular} & 2.55 & 6.99 & 14.4 & 22.3 & 2.91 & 6.77 & 13.9 \\
\hline & & & PCB 138 (pg) & 2.54 & 137967 & 293810 & 425007 & 59247 & 139002 & 251234 \\
\hline & & & \begin{tabular}{|l|} 
PCB $189(\mathrm{pg})$ \\
\end{tabular} & 2.49 & 975 & 2094 & 3025 & 417 & 983 & 1828 \\
\hline & & & \begin{tabular}{|l} 
Organic arsenic $(\mu \mathrm{g})$ \\
\end{tabular} & 2.39 & 149 & 332 & 529 & 54.6 & 142 & 316 \\
\hline & & & \begin{tabular}{|l} 
Methyl mercury $(\mu \mathrm{g})$ \\
\end{tabular} & 2.34 & 4.88 & 10.9 & 18.2 & 1.82 & 5.45 & 9.99 \\
\hline & & & PCB 118 (pg) & 2.31 & 74621 & 157596 & 220450 & 32575 & 74781 & 137130 \\
\hline & & & PCB 180 (pg) & 2.19 & 67873 & 143174 & 207719 & 29689 & 69185 & 124568 \\
\hline & & & PCB 156 (pg) & 2.11 & 9587 & 20271 & 28908 & 4225 & 9708 & 17959 \\
\hline
\end{tabular}




\begin{tabular}{|c|c|c|c|c|c|c|c|c|c|c|}
\hline \multicolumn{2}{|c|}{ Individuals Group } & \multicolumn{3}{|c|}{ Mixtures } & \multicolumn{3}{|c|}{ Cluster exposure } & \multicolumn{3}{|c|}{ Population exposure } \\
\hline Cluster & Description & Major Mixtures & Major substances (unit) & \%Subst & Mean & P95 & P99 & Mean & P95 & P99 \\
\hline & & \multirow{25}{*}{ Mixt-3 (9.7 \%) } & PFTeDA (ng) & 6.45 & 0.03 & 0.17 & 0.24 & 0.008 & 0.03 & 0.12 \\
\hline & & & Benzo[k]fluoranthene (ng) & 6.43 & 16.0 & 44.74 & 64.89 & 7.67 & 16.2 & 38.4 \\
\hline & & & Benzo[b]fluoranthene (ng) & 6.39 & 41.7 & 114 & 171 & 20.1 & 42.1 & 98.6 \\
\hline & & & Benzo[j]fluoranthene (ng) & 6.23 & 20.4 & 53.38 & 80.12 & 9.92 & 21.4 & 49.0 \\
\hline & & & Dibenzo[a,i]pyrene (ng) & 6.06 & 0.19 & 0.61 & 0.88 & 0.10 & 0.26 & 0.53 \\
\hline & & & PFDoA (ng) & 5.40 & 0.25 & 1.09 & 1.54 & 0.07 & 0.21 & 0.83 \\
\hline & & & Chrysene (ng) & 5.12 & 84.0 & 206 & 285 & 42.3 & 89.8 & 192 \\
\hline & & & Benzo[a]anthracene (ng) & 4.21 & 34.3 & 81.58 & 114 & 18.2 & 40.4 & 77.0 \\
\hline & & & Dibenzo[a,e]pyrene (ng) & 4.08 & 1.47 & 3.64 & 5.40 & 0.80 & 1.79 & 3.51 \\
\hline & & & Benzo[a]pyrene (ng) & 3.90 & 17.2 & 37.87 & 50.81 & 9.58 & 20.1 & 37.0 \\
\hline & & & Silver $(\mu \mathrm{g})$ & 3.54 & 167 & 370 & 496 & 108 & 209 & 370 \\
\hline & & & Indeno[1,2,3-cd]pyrene (ng) & 3.22 & 19.0 & 41.03 & 54.89 & 11.4 & 23.2 & 39.5 \\
\hline & & & Dibenzo[a,h]anthracene (ng) & 2.83 & 5.06 & 11.18 & 15.20 & 3.14 & 6.56 & 11.4 \\
\hline & & & HBCD beta (ng) & 2.33 & 0.73 & 1.55 & 2.33 & 0.37 & 0.80 & 1.53 \\
\hline & & & PFDA (ng) & 2.33 & 1.52 & 5.22 & 17.52 & 0.66 & 2.75 & 8.70 \\
\hline & & & Dibenzo[a,i]pyrene (ng) & 2.22 & 0.32 & 1.26 & 2.49 & 0.20 & 0.72 & 1.39 \\
\hline & & & PFOS (ng) & 2.09 & 9.90 & 23.8 & 39.3 & 4.27 & 10.4 & 22.9 \\
\hline & & & Benzo[c]fluorene (ng) & 1.96 & 5.46 & 13.5 & 19.5 & 2.60 & 6.39 & 12.8 \\
\hline & & & Organic arsenic ( $\mu \mathrm{g})$ & 1.80 & 149 & 332 & 529 & 55 & 142 & 316 \\
\hline & & & PFNA (ng) & 1.70 & 0.41 & 1.45 & 3.44 & 0.22 & 0.74 & 2.10 \\
\hline & & & Benzo[g,h,i]perylene (ng) & 1.69 & 38.6 & 77.2 & 99.8 & 25.5 & 50.8 & 81.9 \\
\hline & & & Cadmium ( $\mu \mathrm{g})$ & 1.59 & 19.8 & 41.0 & 59.7 & 13.4 & 25.0 & 44.8 \\
\hline & & & Fluoranthene (ng) & 1.50 & 326 & 688 & 1011 & 190 & 377 & 683 \\
\hline & & & HBCD gamma (ng) & 1.08 & 1.31 & 2.57 & 4.25 & 0.61 & 1.34 & 2.62 \\
\hline & & & OCDD (pg) & 1.03 & 182 & 401 & 659 & 105 & 218 & 418 \\
\hline & & & Myclobutanil (ug) & 5.26 & 1.44 & 3.06 & 3.17 & 0.20 & 0.70 & 251 \\
\hline & & & Fenhexamid $(\mu \mathrm{g})$ & 5.20 & 23.5 & 51.0 & 52.1 & 3.17 & 14.6 & 39.0 \\
\hline & & & Triadimenol ( $\mu \mathrm{g})$ & 5.05 & 1.44 & 2.99 & 3.17 & 0.20 & 0.56 & 2.94 \\
\hline & $\mathrm{N}=1,080$ & & Etofenprox $(\mu \mathrm{g})$ & 4.99 & 1.00 & 2.10 & 2.10 & 0.13 & 0.29 & 2.10 \\
\hline & & & Trifloxystrobin ( $\mu \mathrm{g})$ & 4.99 & 0.07 & 0.14 & 0.14 & 0.009 & 0.02 & 0.14 \\
\hline & & & Quinoxyfen ( $\mu \mathrm{g})$ & 4.99 & 0.62 & 1.31 & 1.31 & 0.08 & 0.18 & 1.31 \\
\hline & & & Spiroxamine $(\mu \mathrm{g})$ & 4.99 & 0.09 & 0.19 & 0.19 & 0.01 & 0.03 & 0.19 \\
\hline & & & Cyfluthrin ( $\mu \mathrm{g})$ & 4.99 & 0.06 & 0.12 & 0.12 & 0.007 & 0.02 & 0.12 \\
\hline & Age $=31.4^{*}$ & & Tetraconazole $(\mu \mathrm{g})$ & 4.99 & 0.06 & 0.12 & 0.12 & 0.007 & 0.02 & 0.12 \\
\hline & {$\left[\mathrm{P}_{2.5} ; \mathrm{P}_{97.5}\right]=[20.9 ; 41.6]$} & & Tebufenpyrad ( $\mu \mathrm{g})$ & 4.99 & 0.02 & 0.05 & 0.05 & 0.003 & 0.007 & 0.05 \\
\hline & & Doct & Methomyl ( $\mu \mathrm{g})$ & 4.50 & 0.04 & 0.08 & 0.08 & 0.005 & 0.018 & 0.07 \\
\hline & & eSt & Teflubenzuron $(\mu \mathrm{g})$ & 4.27 & 0.62 & 1.36 & 1.36 & 0.10 & 0.36 & 1.26 \\
\hline & & & Pyrimethanil $(\mu \mathrm{g})$ & 4.13 & 7.90 & 15.7 & 19.5 & 1.80 & 6.66 & 13.7 \\
\hline & & & Cyprodinyl ( $\mu \mathrm{g})$ & 3.89 & 9.71 & 19.3 & 25.1 & 2.16 & 7.68 & 15.2 \\
\hline & BMI $=23.6$ & & Bifenthrin ( $\mu \mathrm{g})$ & 3.79 & 0.73 & 1.48 & 1.98 & 0.18 & 0.65 & 1.23 \\
\hline & {$\left[\mathrm{P}_{2.5} ; \mathrm{P}_{97.5}\right]=[20.9 ; 41.6]$} & & Mepanipyrim ( $\mu \mathrm{g})$ & 3.68 & 1.11 & 2.51 & 2.62 & 0.16 & 0.71 & 1.80 \\
\hline & & & Metalaxyl ( $\mu \mathrm{g})$ & 3.50 & 0.90 & 1.79 & 2.22 & 0.21 & 0.77 & 1.43 \\
\hline & & & Penconazole $(\mu \mathrm{g})$ & 2.98 & 0.54 & 1.22 & 1.29 & 0.08 & 0.40 & 0.86 \\
\hline & & & Boscalid $(\mu \mathrm{g})$ & 2.35 & 6.71 & 14.5 & 17.1 & 1.38 & 4.73 & 10.9 \\
\hline & & & Iprodione ( $\mu \mathrm{g})$ & 1.87 & 31.1 & 69.1 & 115 & 10.5 & 36.3 & 66.1 \\
\hline & WeightGain $=13.0$ & & Chlorpyrifos-ethyl ( $\mu \mathrm{g})$ & 1.81 & 5.14 & 10.6 & 11.1 & 1.13 & 4.92 & 8.94 \\
\hline & {$\left[\mathrm{P}_{2.5} ; \mathrm{P}_{97.5}\right]=[2.0 ; 25.0]$} & & Lambda-cyhalothrin $(\mu \mathrm{g})$ & 1.32 & 1.15 & 2.56 & 4.46 & 0.37 & 1.32 & 2.15 \\
\hline
\end{tabular}




\begin{tabular}{|c|c|c|c|c|c|c|c|c|c|c|}
\hline \multicolumn{2}{|c|}{ Individuals Group } & \multicolumn{3}{|c|}{ Mixtures } & \multicolumn{3}{|c|}{ Cluster exposure } & \multicolumn{3}{|c|}{ Population exposure } \\
\hline Cluster & Description & Major Mixtures & Major substances (unit) & \%Subst & Mean & P95 & P99 & Mean & P95 & P99 \\
\hline & & & Procymidone $(\mu \mathrm{g})$ & 1.26 & 5.31 & 11.6 & 16.9 & 1.71 & 5.82 & 9.87 \\
\hline & & & \begin{tabular}{|l|} 
Azoxystrobin $(\mu \mathrm{g})$ \\
\end{tabular} & 1.18 & 1.32 & 2.94 & 4.18 & 0.27 & 0.81 & 2.71 \\
\hline & & & Chlorpyrifos-methyl $(\mu \mathrm{g})$ & 1.17 & 0.72 & 1.46 & 2.71 & 0.38 & 0.80 & 1.19 \\
\hline & & & Chromium VI ( $\mu \mathrm{g})$ & 2.31 & 83.6 & 184 & 322 & 55.5 & 98.9 & 150 \\
\hline & & & \begin{tabular}{|l|} 
Chromium III $(\mu \mathrm{g})$ \\
\end{tabular} & 2.19 & 578 & 1528 & 2767 & 338 & 626 & 1041 \\
\hline & & & \begin{tabular}{|l} 
Lead $(\mu \mathrm{g})$ \\
\end{tabular} & 2.17 & 26.0 & 60.8 & 109 & 15.7 & 27.9 & 44.7 \\
\hline & & & \begin{tabular}{|l|} 
Cobalt $(\mu \mathrm{g})$ \\
\end{tabular} & 2.17 & 27.4 & 70.7 & 126 & 15.8 & 28.8 & 46.2 \\
\hline & & & \begin{tabular}{|l|} 
Barium $(\mu \mathrm{g})$ \\
\end{tabular} & 2.08 & 1104 & 2673 & 4704 & 653 & 1203 & 1975 \\
\hline & & & \begin{tabular}{|l} 
Tellurium $(\mu \mathrm{g})$ \\
\end{tabular} & 2.08 & 3.36 & 7.94 & 11.9 & 2.36 & 4.25 & 6.19 \\
\hline & & & \begin{tabular}{|l} 
Vanadium $(\mu \mathrm{g})$ \\
\end{tabular} & 2.06 & 121 & 282 & 466 & 77.9 & 147 & 224 \\
\hline & & & \begin{tabular}{|l} 
Nickel $(\mu \mathrm{g})$ \\
\end{tabular} & 2.05 & 364 & 865 & 1467 & 194 & 361 & 601 \\
\hline & & & Inorganic arsenic $(\mu \mathrm{g})$ & 1.95 & 50.7 & 120 & 210 & 31.9 & 60.8 & 95.8 \\
\hline & & & \begin{tabular}{|l|} 
Antimony $(\mu \mathrm{g})$ \\
\end{tabular} & 1.87 & 3.45 & 9.04 & 15.7 & 2.13 & 4.20 & 6.94 \\
\hline & & & \begin{tabular}{|l} 
HCDF $123678(\mathrm{pg})$ \\
\end{tabular} & 1.86 & 8.38 & 24.5 & 54.4 & 5.16 & 9.74 & 15.9 \\
\hline & & & \begin{tabular}{|l|} 
Pyrene (ng) \\
\end{tabular} & 1.85 & 765 & 2354 & 5244 & 482 & 919 & 1469 \\
\hline & & TE-F-PAH (199\%) & \begin{tabular}{|l|} 
Aluminium $(\mu \mathrm{g})$ \\
\end{tabular} & 1.83 & 6255 & 16044 & 31045 & 3591 & 7017 & 12051 \\
\hline & & & \begin{tabular}{|l|} 
HCDF $123478(\mathrm{pg})$ \\
\end{tabular} & 1.80 & 14.7 & 43.8 & 107 & 8.79 & 16.4 & 28.2 \\
\hline & & & HCDF 1234789 (pg) & 1.80 & 10.3 & 31.9 & 75.0 & 6.22 & 11.6 & 19.6 \\
\hline & & & \begin{tabular}{|l|} 
OCDF (pg) \\
\end{tabular} & 1.77 & 51.1 & 159 & 405 & 30.5 & 57.3 & 94.6 \\
\hline & & & \begin{tabular}{|l|} 
Lithium $(\mu \mathrm{g})$ \\
\end{tabular} & 1.74 & 80.2 & 169 & 257 & 53.0 & 103 & 145 \\
\hline & & & \begin{tabular}{|l|} 
HCDF $123789(\mathrm{pg})$ \\
\end{tabular} & 1.73 & 2.18 & 6.63 & 16.8 & 1.30 & 2.50 & 4.11 \\
\hline & & & \begin{tabular}{|l|} 
HCDD $123678(\mathrm{pg})$ \\
\end{tabular} & 1.71 & 12.1 & 37.1 & 88.2 & 7.22 & 14.0 & 24.0 \\
\hline & & & \begin{tabular}{|l|} 
HCDD $123789(\mathrm{pg})$ \\
\end{tabular} & 1.70 & 4.09 & 12.3 & 29.8 & 2.50 & 4.87 & 7.81 \\
\hline & & & HCDF $1234678(\mathrm{pg})$ & 1.69 & 19.2 & 57.9 & 147 & 11.2 & 21.1 & 37.3 \\
\hline & & & HCDF $234678(\mathrm{pg})$ & 1.68 & 4.21 & 12.7 & 30.9 & 2.46 & 4.57 & 7.91 \\
\hline & & & \begin{tabular}{|l|} 
HCDD $123478(\mathrm{pg})$ \\
\end{tabular} & 1.65 & 3.15 & 9.12 & 23.0 & 1.90 & 3.74 & 6.35 \\
\hline & & & \begin{tabular}{|l} 
Cadmium $(\mu \mathrm{g})$ \\
\end{tabular} & 1.65 & 24.7 & 68.2 & 148 & 13.4 & 25.0 & 44.8 \\
\hline & & & Zearalenone (ng) & 1.60 & 727 & 2029 & 4184 & 489 & 983 & 1619 \\
\hline & & & & & & & & & & \\
\hline & & & Bupirimate $(\mu \mathrm{g})$ & 7.90 & 2.75 & 6.63 & 7.64 & 0.44 & 1.20 & 6.52 \\
\hline & & & \begin{tabular}{|l} 
Dichlorvos ( $\mu \mathrm{g})$ \\
\end{tabular} & 7.87 & 0.11 & 0.25 & 0.29 & 0.02 & 0.05 & 0.25 \\
\hline & & & \begin{tabular}{|l} 
Endosulfan $(\mu \mathrm{g})$ \\
\end{tabular} & 7.84 & 1.67 & 4.05 & 4.49 & 0.27 & 0.73 & 3.90 \\
\hline & & & \begin{tabular}{|l|} 
Acrinathrin $(\mu \mathrm{g})$ \\
\end{tabular} & 7.83 & 0.83 & 1.89 & 2.31 & 0.14 & 0.57 & 1.86 \\
\hline & & & \begin{tabular}{|l} 
Kresoxim-methyl $(\mu \mathrm{g})$ \\
\end{tabular} & 7.65 & 0.22 & 0.53 & 0.59 & 0.03 & 0.10 & 0.51 \\
\hline & & & \begin{tabular}{|l|} 
Azoxystrobin $(\mu \mathrm{g})$ \\
\end{tabular} & 7.05 & 1.32 & 2.94 & 4.18 & 0.27 & 0.81 & 2.71 \\
\hline & & & \begin{tabular}{|l|} 
Penconazole $(\mu \mathrm{g})$ \\
\end{tabular} & 5.20 & 0.54 & 1.22 & 1.29 & 0.08 & 0.40 & 0.86 \\
\hline & & & Boscalid ( $\mu \mathrm{g})$ & 4.66 & 6.71 & 14.5 & 17.1 & 1.38 & 4.73 & 10.9 \\
\hline & & & \begin{tabular}{|l|} 
Tebuconazole $(\mu \mathrm{g})$ \\
\end{tabular} & 4.51 & 0.29 & 0.57 & 0.58 & 0.06 & 0.29 & 0.56 \\
\hline & & Pest-2 (18.6 \%) & \begin{tabular}{|l} 
Dimethoate $(\mu \mathrm{g})$ \\
\end{tabular} & 4.44 & 3.71 & 7.21 & 7.29 & 0.77 & 3.61 & 7.19 \\
\hline & & & \begin{tabular}{|l} 
Fenbuconazole $(\mu \mathrm{g})$ \\
\end{tabular} & 4.42 & 0.70 & 1.36 & 1.36 & 0.14 & 0.68 & 1.36 \\
\hline & & & \begin{tabular}{|l|} 
Mepanipyrim $(\mu \mathrm{g})$ \\
\end{tabular} & 4.11 & 1.11 & 2.51 & 2.62 & 0.16 & 0.71 & 1.80 \\
\hline & & & \begin{tabular}{|l|} 
Procymidone $(\mu \mathrm{g})$ \\
\end{tabular} & 3.79 & 5.31 & 11.6 & 16.9 & 1.71 & 5.82 & 9.87 \\
\hline & & & \begin{tabular}{|l|} 
Phosmet $(\mu \mathrm{g})$ \\
\end{tabular} & 2.85 & 1.06 & 2.39 & 2.39 & 0.28 & 1.20 & 1.96 \\
\hline & & & \begin{tabular}{|l|l|l|l} 
Chlorpyrifos-ethyl $(\mu \mathrm{g})$ \\
\end{tabular} & 2.60 & 5.14 & 10.6 & 11.1 & 1.13 & 4.92 & 8.94 \\
\hline & & & \begin{tabular}{|l|} 
Metalaxyl $(\mu \mathrm{g})$ \\
\end{tabular} & 2.53 & 0.90 & 1.79 & 2.22 & 0.21 & 0.77 & 1.43 \\
\hline & & & \begin{tabular}{|l} 
Lambda-cyhalothrin $(\mu \mathrm{g})$ \\
\end{tabular} & 2.36 & 1.15 & 2.56 & 4.46 & 0.37 & 1.32 & 2.15 \\
\hline & & & \begin{tabular}{|l} 
Cyprodinyl $(\mu \mathrm{g})$ \\
\end{tabular} & 2.00 & 9.71 & 19.3 & 25.1 & 2.16 & 7.68 & 15.2 \\
\hline & & & \begin{tabular}{|l} 
Fludioxonil $(\mu \mathrm{g})$ \\
\end{tabular} & 1.53 & 10.0 & 24.4 & 37.2 & 3.58 & 12.2 & 22.4 \\
\hline
\end{tabular}




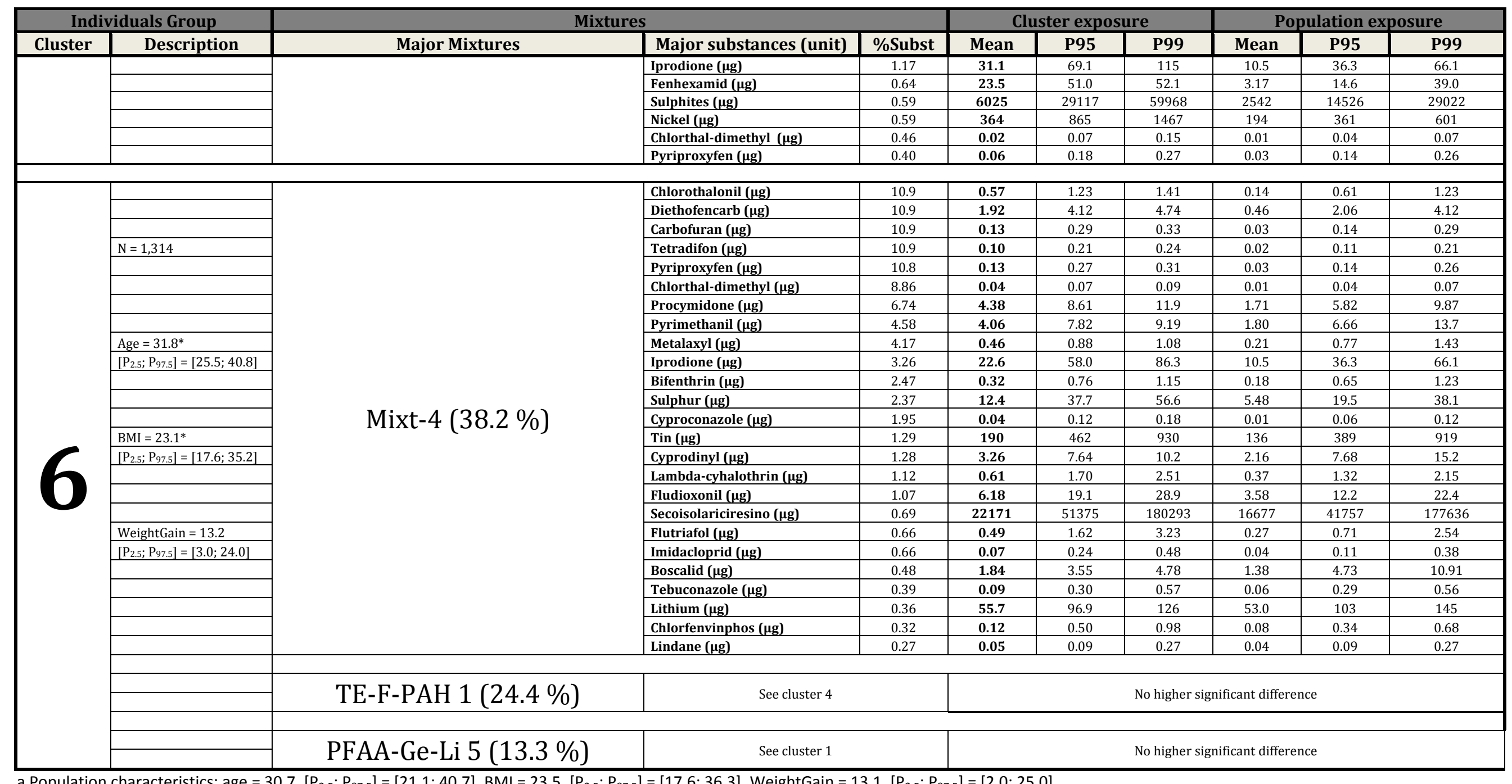




\section{Discussion}

The application of the SNMU and the hierarchical clustering method to the exposure datasets from the EDEN and ELFE cohorts and TDS 2 enabled us to identify the eight main chemical mixtures to which pregnant women in France are exposed before and during pregnancy. The method also made it possible to define groups of women with specific co-exposure profiles. Thus, six groups of pregnant women associated with the eight mixtures were identified. Except for specific compounds, similar mixtures were obtained concerning the periods before and during pregnancy using the EDEN survey. Moreover, these similar mixtures were also found from the ELFE cohort exposure data during pregnancy.

\section{The SNMU and the choice of number of mixtures $K$}

The choice of an optimal number of exposure systems $K$ remains a major challenge. There is no theoretical result for determining this number in general, but different approaches have been proposed. Thus, in Bayesian NMF, Cemgil (2009) and Schmidt et al. (2009) evaluated a marginal likelihood for each candidate value of $K$ and the value with highest marginal likelihood is considered as the optimal choice. Mørup and Hansen (2009), Yang et al. (2010) and Tan and Févotte (2013) also proposed methods in which the number of exposure systems is set to a large value and nonpertinent exposure systems are driven to zero during simulation. With the SNMU method, this problem is solved in part. This is because the recursive algorithm used allows us to identify exposure systems one by one. From the original exposure matrix $E=R_{1}$, the first approximation $W_{: 1} H_{1}$ : is extracted and therefore subtracted from this matrix, i.e. $R_{2}=R_{1}-W_{: 1} H_{1:}$. The same procedure is thus applied on the new obtained matrix $R_{2}$. In this way, another approximation is extracted corresponding to the first approximation for the matrix $R_{2}$ and to the second approximation for the original exposure matrix $E$. At each step $k$, an approximation $W_{: k} H_{k}$ is extracted from a new residual matrix $R_{k}$. It corresponds to the $k^{s t}$ approximation of the original exposure matrix $E$ and is identical, regardless of the number of exposure systems. Hence, the first approximations extracted, regardless 
of the number of exposure systems, are identical. When applying the clustering method to the matrix $H$ obtained with this procedure, the $K$ values for which one or more exposure systems were not used to characterize a cluster were rejected. Therefore, the optimal number of $K$ was defined as the largest of the non-rejected values. In this study, eight exposure systems were extracted on each exposure dataset and led to division of the overall study population into six clusters characterised by these exposure systems.

\section{The sparsity coefficient and its bounds}

As described by Gillis and Plemmons (2013), the SNMU requires human supervision to determine the sparsity coefficient and its bounds. No mathematical criterion to automatically determine these values is available. The values depend only on the degree of sparsity needed by the user to reduce the dataset. In our case study, we decided to set the bounds to 0.2 and 0.99 to make $\lambda$ vary on a large range of values and we observed that results remained stable. We also observed that when $k$ increased, the additional exposure systems were more sparse and the first exposure system was always less sparse. We set the coefficient lambda to high sparsity for the first system to force it to be sparse, whereas the values for the other $k$ were set lower.

\section{Spatial and temporal mixture comparison}

The EDEN and ELFE cohorts were selected to identify main mixtures to which pregnant women in France were exposed during pregnancy. These two cohorts are the most recent and complete surveys available for this population. EDEN was the first survey conducted in a local area, whereas ELFE is a national survey including a large number of women. The FFQ used in the ELFE study was a modified version from the EDEN study. Some differences were observed, in particular on the number of food items: 137 in EDEN and 125 in ELFE. Some food items were considered individually in EDEN and grouped together in ELFE. For example, in the EDEN study, food items like "red and rosé wine", "white wine", "beer", "cider", "lemonade", "light soda" and "light cola" were grouped in the ELFE study respectively in food items "white, red or rosé wine", "cider and beer" and "light lemonade and soda". Likewise, "fresh cream", "butter" and "margarine", light and non-light, were considered 
individually in the EDEN study, whereas in the ELFE study, consumption of "fresh cream", "butter" and "margarine" were recorded. Despite the differences in survey periods (2003-2006 for EDEN vs from 2011 for ELFE), regions (2 regions vs mainland France) and in the FFQs, comparable mixtures were obtained.

Similar results were also obtained on clustering from EDEN before and during pregnancy exposure data. The study population was divided into six clusters for each exposure data. Individuals in five clusters among the six were significantly more exposed to substances from mixtures characterising the clusters.

\section{Comparison of mixture before and during pregnancy}

Seven of the eight mixtures identified before and during pregnancy from EDEN exposures presented similarities and six of them were also observed in ELFE during pregnancy. In EDEN before pregnancy, "Mixt-1" mixture consisting of certain heat-induced contaminants (acrylamide and six PAHS), some mycotoxins, pesticides, phytoestrogens and additives was not identified from EDEN during pregnancy. This can be explained by the fact that the common foods contributing to exposure to these substances were coffee, cereal products (bread products, pasta, aperitif biscuits, semolina, etc.), potato products, meat and delicatessen meats, crustaceans and molluscs, fruit juices, milk and beans (Bemrah et al., 2012; Nougadère et al., 2012; Sirot et al., 2012a; Chan-Hon-Tong et al., 2013; Sirot et al., 2013; Veyrand et al., 2013). Consumption of coffee, meat and delicatessen meats, crustaceans and molluscs were reduced during pregnancy by the EDEN pregnant women population (Chan-Hon-Tong et al., 2013). This consumption reduction was also observed in pregnant women in Portugal (Pinto et al., 2009) for red meat, coffee and tea. In EDEN during pregnancy, "Mixt-2" mixture consisting mainly of DL-PCB, NDL-PCB, BFR and PAH compounds was not identified from EDEN before pregnancy. This result can be explained by the fact that the main food contributors to the exposure to these substances were fish products, butter, cheese, crustaceans and molluscs, cereal products, milk, meat and delicatessen meats (Sirot et al., 2012b; Chan-Hon-Tong et al., 2013; Veyrand et al., 2013; Rivière et al., 2014), even though consumption of these last food groups was 
reduced during pregnancy. During pregnancy, women increased their consumption of fish, butter, milk, cheese and cereal products (Chan-Hon-Tong et al., 2013). This trend concerning the consumption of fish, milk and cereal products (especially bread) was also observed in pregnant women in Portugal (Pinto et al., 2009).

\section{Comparison with the general adult population}

The NMF method was also used by Traoré et al. (2016) to identify the main mixtures to which the general adult population in France is exposed through diet. In this study, dietary patterns were first defined using the LS-NMF method (Wang et al., 2006), a modified version of NMF (Lee and Seung, 2001) and solved by a multiplicative algorithm based on gradient descent, on individual consumption data, connected with mixtures and associated with individual clusters. In the present study, the SNMU method, solved by a recursive algorithm based on Lagrangian relaxation, was applied to exposure data concerning pregnant women in France to define mixtures. Despite the above differences, two mixtures identified from the three exposure datasets and one from ELFE during pregnancy in the present study presented some similarities with three mixtures identified in the general adult population in France by Traoré et al. (2016). These mixtures consisting of trace elements, PAHs and pesticides were associated with the "Mediterranean" diet which is mostly eaten by women. This comparison consolidates the method's robustness since the consumptions were recorded using FFQ in the EDEN and ELFE studies, whereas in INCA 2, a seven-day record was used. The mixtures associated with the "Traditional", the "Snacking" and the "Simplicity" diets in Traoré et al. (2016) were not found in the pregnant women cohorts, which could be explained by a reduction of the consumption of the associated foods during pregnancy.

\section{Uncertainty Sources}

Several sources of uncertainty may affect these results.

An FFQ record was considered for each woman. In EDEN, the first FFQ was collected at inclusion and concerned the diet in the year before pregnancy, and the second FFQ at delivery and concerned the diet during the third trimester of pregnancy. In ELFE, the FFQ was recorded at delivery and 
described the dietary habits during the third trimester of pregnancy. Although the FFQ approach made it possible to collect information on a large number of individuals at lower cost, the consumed quantity is generally overestimated compared with other methods of recording (Rothenberg, 2009), especially when the number of foods recorded is high (Kim and Holowaty, 2003). This may have affected the exposures, which are probably over-estimated, and ultimately, the mixture compositions. Another issue concerning the populations in these cohorts is under-reporting by individuals. As recommended by the European Food Safety Authority (EFSA, 2014), under-reporting individuals, i.e. individuals who under-reported their consumption frequencies, were included in this study. This means that no exclusion on daily energy intake was applied (i.e. individuals with daily energy intake lower than the $3^{\text {rd }}$ percentile or higher than the $97^{\text {th }}$ percentile) and that may have affected the identification of the mixtures and the clusters with similar exposure profiles.

Another potential source of uncertainty is related to the concentration data collected using the TDS approach. As in any survey, TDS provided a snapshot of the general contamination of the population diet at a specific point in time. However, concentration data change over time, especially for pesticides and additives which constant changing regulations, but also for trace elements as observed for arsenic and lead when comparing TDS 1 (Leblanc et al., 2005) and TDS 2 (Arnich et al., 2012) in France. For example, due to the prohibition on lead in automotive gasolines, but also the reduction of lead presence in water pipes and interior paints, the exposure level is decreasing in industrialised countries (Etchevers et al., 2014) such as France. Climate change or different weather conditions could also have an effect on concentrations of mycotoxins as observed by Cano-Sancho et al. (2012) when compared European products with ethnical food such as Mexican corn-based foods. Therefore, regarding concentration data, the present mixtures come from observations over the period 2007-2009 (the TDS 2 sampling period) and should be updated with more recent data. Moreover, even though TDS 2 covered a large range of substances, some substances that may contribute to real-life mixtures were not analysed and the mixture could be incomplete. For example, substances such as phthalates, alkyl phenols and furane were not analysed in TDS 2 whereas they 
were analysed during the French infant TDS (Hulin et al., 2014). However, the TDS protocol is based on previous experience of food contamination and should cover the major substances present in food. Also, some foods like exotic fruits, which were recorded in the EDEN and ELFE cohorts were not analysed in TDS 2 as they are not largely consumed by the general population, and this could lead to a slight underestimation of exposure to the substances present in these products. Dilution due to the pooling of samples is one of the issues in TDS. Actual values in one sample may be diluted below the LOD in composite samples and lead to censored data. The use of the lower bound (LB) scenario, i.e. undetected values were set to 0 and detected but unquantified values to the limit of detection, could lead to the exclusion of substances that could be present in a food item and to underestimation of exposure. Then lower analytical limits would be useful to precise the exposure to chemicals with a high censorship rate.

The mixtures were identified using chronic exposure assessment, as TDS data could not be used for acute exposure assessment. However, for some substances, the time frame related to acute exposure may be more appropriate for certain toxicological effects. As a result, it would be interesting to apply this approach to acute exposure data estimated with national monitoring programmes as was done for the general population in France (Crépet et al., 2013).

Moreover, it would be interesting to investigate other sources of exposure such as air or dust. For instance, it is possible that the occupational environment of pregnant women contributed to exposure. Finally, as this approach was based on external exposure, it may be useful to apply this method to internal exposure data for a large range of substances.

\section{Conclusion}

This study allowed us to point out the diversity of mixtures to which pregnant women in France are exposed before and during pregnancy through diet. It also showed new avenues for research and where efforts are to be made to prioritise combinations of substances for which it is essential to investigate possible combined toxicological effects. These mixtures can be used in epidemiology to study the association with health effects in children. 


\section{Funding sources}

This research was supported by the French Agence Nationale de la Recherche (ANR) (Grant ANR14-CE21-0002-02).

\section{Acknowledgments}

- The EDEN cohort is supported by grants from FRM, Inserm, IReSP, Nestlé, the French Ministry of Health, ANR, Univ. Paris-Sud, InVS, ANSES and MGEN. The funding source had no involvement in study design, in the collection, analysis, and interpretation of data, in the writing of the report, or in the decision to submit the paper for publication. The study received approval from the Ethics Committee of Kremlin-Bicêtre University Hospital and from the French National Commission for Data Protection (CNIL). The EDEN mother-child cohort study group includes: I. Annesi-Maesano, J.Y. Bernard, J. Botton, M-A. Charles, P. Dargent-Molina, B. de Lauzon-Guillain, P. Ducimetière, M. de Agostini, B. Foliguet, A. Forhan, X. Fritel, A. Germa, V. Goua, R. Hankard, B. Heude, M. Kaminski, B. Larroque, N. Lelong, J. Lepeule, G. Magnin, L. Marchand, C. Nabet, F. Pierre, R. Slama, M.J. SaurelCubizolles, M. Schweitzer, O. Thiebaugeorges.

- ELFE is a study conducted jointly by the National Institute of Demographic Studies (Ined), the French National Institute for Health and Medical Research (Inserm), French blood establishment (EFS), French Institute for Public Health Surveillance (InVS), French National Institute for Statistics and Economic Studies (Insee), General Directorate for Health (DGS, Ministry of Health), General Directorate for Risk Prevention (DGPR, Ministry of Environment), Directorate for Research, Studies, Evaluation and Statistics (Drees), and the French National Family Allowance Fund (Cnaf). It benefits from additional funding from the Ministry of Research, Committee on SHS data (CCDSHS) and Ministry of Culture and 
Communication (Deps). As part of the RECONAI platform, ELFE benefits from ANR funding (ANR-11-EQPX-0038). 


\section{References:}

Arnich, N., Sirot, V., Rivière, G., Jean, J., Noël, L., Guérin, T., and Leblanc, J. C. (2012). Dietary exposure to trace elements and health risk assessment in the 2nd French Total Diet Study. [Article]. Food and Chemical Toxicology, 50(7), 2432-2449. doi: 10.1016/j.fct.2012.04.016

Béchaux, C., Zetlaoui, M., Tressou, J., Leblanc, J. C., Héraud, F., and Crépet, A. (2013). Identification of pesticide mixtures and connection between combined exposure and diet. Food and Chemical Toxicology, 59, 191-198. doi: 10.1016/j.fct.2013.06.006

Bemrah, N., Jean, J., Rivière, G., Sanaa, M., Leconte, S., Bachelot, M., Deceuninck, Y., Bizec, B. L., Dauchy, X., Roudot, A. C., Camel, V., Grob, K., Feidt, C., Picard-Hagen, N., Badot, P. M., Foures, F., and Leblanc, J. C. (2014). Assessment of dietary exposure to bisphenol A in the French population with a special focus on risk characterisation for pregnant French women. Food and Chemical Toxicology, 72, 90-97. doi: 10.1016/j.fct.2014.07.005

Bemrah, N., Vin, K., Sirot, V., Aguilar, F., Ladrat, A. C., Ducasse, C., Gey, J. L., Rétho, C., Nougadere, A., and Leblanc, J. C. (2012). Assessment of dietary exposure to annatto (E160b), nitrites (E249250), sulphites (E220-228) and tartaric acid (E334) in the French population: The second French total diet study. Food Additives and Contaminants - Part A Chemistry, Analysis, Control, Exposure and Risk Assessment, 29(6), 875-885. doi: 10.1080/19440049.2012.658525

Canfield, R. L., Henderson, C. R., Cory-Slechta, D. A., Cox, C., Jusko, T. A., and Lanphear, B. P. (2003). Intellectual Impairment in Children with Blood Lead Concentrations below $10 \mu \mathrm{g}$ per Deciliter. The New England journal of medicine, 348(16), 1517-1526. doi: 10.1056/NEJMoa022848

Cano-Sancho, G., Ramos, A. J., Marín, S., and Sanchis, V. (2012). Occurrence of fumonisins in Catalonia (Spain) and an exposure assessment of specific population groups. [Article]. Food Additives and Contaminants - Part A Chemistry, Analysis, Control, Exposure and Risk Assessment, 29(5), 799-808. doi: 10.1080/19440049.2011.644813

Cemgil, A. T. (2009). Bayesian inference for nonnegative matrix factorisation models. [Article]. Computational Intelligence and Neuroscience, 2009. doi: 10.1155/2009/785152

Chan-Hon-Tong, A., Charles, M. A., Forhan, A., Heude, B., and Sirot, V. (2013). Exposure to food contaminants during pregnancy. [Article]. Science of the Total Environment, 458-460, 27-35. doi: 10.1016/j.scitotenv.2013.03.100

Claus Henn, B., Coull, B. A., and Wright, R. O. (2014). Chemical mixtures and children's health. Current Opinion in Pediatrics, 26(2), 223-229. doi: 10.1097/mop.0000000000000067

Crépet, A., and Tressou, J. (2011). Bayesian nonparametric model for clustering individual coexposure to pesticides found in the French diet. Bayesian Analysis, 6(1), 127-144. doi: 10.1214/11-BA604

Crépet, A., Tressou, J., Graillot, V., Béchaux, C., Pierlot, S., Héraud, F., and Leblanc, J. C. (2013). Identification of the main pesticide residue mixtures to which the French population is exposed. Environmental Research, 126, 125-133. doi: 10.1016/j.envres.2013.03.008

Czarnota, J., Gennings, C., Colt, J. S., De Roos, A. J., Cerhan, J. R., Severson, R. K., Hartge, P., Ward, M. H., and Wheeler, D. C. (2015). Analysis of Environmental Chemical Mixtures and Non-Hodgkin Lymphoma Risk in the NCl-SEER NHL Study. Environmental Health Perspectives. doi: :10.1289/ehp.1408630

Deschamps, V., de Lauzon-Guillain, B., Lafay, L., Borys, J. M., Charles, M. A., and Romon, M. (2009). Reproducibility and relative validity of a food-frequency questionnaire among French adults and adolescents. European Journal of Clinical Nutrition, 63(2), 282-291. doi: 10.1038/sj.ejcn.1602914

Dorea, J. G., and Donangelo, C. M. (2006). Early (in uterus and infant) exposure to mercury and lead. Clinical Nutrition, 25(3), 369-376. doi: 10.1016/j.clnu.2005.10.007

Duarte-Salles, T., von Stedingk, H., Granum, B., Gützkow, K. B., Rydberg, P., Törnqvist, M., Mendez, M. A., Brunborg, G., Brantsæter, A. L., Meltzer, H. M., Alexander, J., and Haugen, M. (2013). 
Dietary acrylamide intake during pregnancy and fetal growth-results from the Norwegian Mother and child cohort study (MoBa). [Article]. Environmental Health Perspectives, 121(3), 374-379. doi: 10.1289/ehp.1205396

Dubuisson, C., Lioret, S., Touvier, M., Dufour, A., Calamassi-Tran, G., Volatier, J. L., and Lafay, L. (2010). Trends in food and nutritional intakes of French adults from 1999 to 2007: Results from the INCA surveys. British Journal of Nutrition, 103(7), 1035-1048. doi: $10.1017 /$ S0007114509992625

EFSA (2013). Scientific Opinion of the Panel on Plant Protection Products and their Residues (PPR) on the identification of pesticides to be included in cumulative assessment groups on the basis of their toxicological profile. EFSA Journal; 11(7):3293. [131 pp.]. doi: 10.2903/j.efsa.2013.3293 (EFSA, Parma, Italy).

EFSA (2014). Guidance on the EU Menu methodology. EFSA Journal; 12(12. [3944-n/a pp.]. doi: 10.2903/j.efsa.2014.3944.

Etchevers, A., Bretin, P., Lecoffre, C., Bidondo, M. L., Le Strat, Y., Glorennec, P., and Le Tertre, A. (2014). Blood lead levels and risk factors in young children in France, 2008-2009. [Article]. International Journal of Hygiene and Environmental Health, 217(4-5), 528-537. doi: 10.1016/j.ijheh.2013.10.002

Gazan, R., Béchaux, C., Crépet, A., Sirot, V., Drouillet-Pinard, P., Dubuisson, C., and Havard, S. (2016). Dietary patterns in the French adult population: a study from the second French national cross-sectional dietary survey (INCA2) (2006-2007). [Article in Press]. British Journal of Nutrition, 1-16. doi: 10.1017/S0007114516001549

GEMS/Food-EURO (2013). Addendum 2013 - Second Workshop on Reliable Evaluation of Low-Level Contamination of Food. Workshop in the frame of GEMS/Food-EURO. Technical report. [pp.]. (WHO, Kulmbach, Germany).

Gillis, N., and Glineur, F. (2010). Using underapproximations for sparse nonnegative matrix factorization. Pattern Recognition, 43(4), 1676-1687. doi: http://dx.doi.org/10.1016/i.patcog.2009.11.013

Gillis, N., and Plemmons, R. J. (2013). Sparse nonnegative matrix underapproximation and its application to hyperspectral image analysis. Linear Algebra and its Applications, 438(10), 3991-4007. doi: http://dx.doi.org/10.1016/j.laa.2012.04.033

Hercberg, S. (2000). Portions alimentaires : manuel photos pour l'estimation des quantités. Paris: Economica.

Hercberg, S., Deheeger, M., and Preziosi, P. (2002). SU.VI.MAX. Portions alimentaires : Manuel photos pour l'estimation des quantités: Paris: BMJ Publishing Group.

Heude, B., Forhan, A., Slama, R., Douhaud, L., Bedel, S., Saurel-Cubizolles, M. J., Hankard, R., Thiebaugeorges, O., De Agostini, M., Annesi-Maesano, I., Kaminski, M., Charles, M. A., Annesi-Maesano, I., Bernard, J. Y., Botton, J., Charles, M. A., Dargent-Molina, P., de LauzonGuillain, B., Ducimetière, P., de Agostini, M., Foliguet, B., Forhan, A., Fritel, X., Germa, A., Goua, V., Hankard, R., Heude, B., Kaminski, M., Larroque, B., Lelong, N., Lepeule, J., Magnin, G., Marchand, L., Nabet, C., Pierre, F., Slama, R., Saurel-Cubizolles, M. J., Schweitzer, M., and Thiebaugeorges, O. (2016). Cohort Profile: The EDEN mother-child cohort on the prenatal and early postnatal determinants of child health and development. [Article]. International Journal of Epidemiology, 45(2), 353-363. doi: 10.1093/ije/dyv151

Hulin, M., Bemrah, N., Nougadère, A., Volatier, J. L., Sirot, V., and Leblanc, J. C. (2014). Assessment of infant exposure to food chemicals: The French Total Diet Study design. Food Additives and Contaminants - Part A Chemistry, Analysis, Control, Exposure and Risk Assessment, 31(7), 1226-1239. doi: 10.1080/19440049.2014.921937

Kim, D. J., and Holowaty, E. J. (2003). Brief, validated survey instruments for the measurement of fruit and vegetable intakes in adults: A review. [Article]. Preventive Medicine, 36(4), 440-447. doi: 10.1016/S0091-7435(02)00040-3 
Lê, S., Josse, J., and Husson, F. (2008). FactoMineR: An R package for multivariate analysis. [Article]. Journal of Statistical Software, 25(1), 1-18.

Leblanc, J. C., Guérin, T., Noël, L., Calamassi-Tran, G., Volatier, J. L., and Verger, P. (2005). Dietary exposure estimates of 18 elements from the 1st French Total Diet Study. [Article]. Food Additives and Contaminants, 22(7), 624-641. doi: 10.1080/02652030500135367

Lee, D. D., and Seung, H. S. (2001). Algorithms for non-negative matrix factorization. Paper presented at the Advances in Neural Information Processing Systems.

Lioret, S., Dubuisson, C., Dufour, A., Touvier, M., Calamassi-Tran, G., Maire, B., Volatier, J. L., and Lafay, L. (2010). Trends in food intake in French children from 1999 to 2007: Results from the INCA (étude Individuelle Nationale des Consommations Alimentaires) dietary surveys. British Journal of Nutrition, 103(4), 585-601. doi: 10.1017/S0007114509992078

Mendez, M. A., Garcia-Esteban, R., Guxens, M., Vrijheid, M., Kogevinas, M., Goñi, F., Fochs, S., and Sunyer, J. (2011). Prenatal organochlorine compound exposure, rapid weight gain, and overweight in infancy. Environmental Health Perspectives, 119(2), 272-278. doi: 10.1289/ehp.1002169

Mørup, M., and Hansen, L. K. (2009). Automatic relevance determination for multi-way models. Journal of Chemometrics, 23(7-8), 352-363. doi: 10.1002/cem.1223

Nougadère, A., Sirot, V., Kadar, A., Fastier, A., Truchot, E., Vergnet, C., Hommet, F., Baylé, J., Gros, P., and Leblanc, J. C. (2012). Total diet study on pesticide residues in France: Levels in food as consumed and chronic dietary risk to consumers. [Article]. Environment International, 45(1), 135-150. doi: 10.1016/j.envint.2012.02.001

Pedersen, M., Von Stedingk, H., Botsivali, M., Agramunt, S., Alexander, J., Brunborg, G., Chatzi, L., Fleming, S., Fthenou, E., Granum, B., Gutzkow, K. B., Hardie, L. J., Knudsen, L. E., Kyrtopoulos, S. A., Mendez, M. A., Merlo, D. F., Nielsen, J. K., Rydberg, P., Segerbäck, D., Sunyer, J., Wright, J., Törnqvist, M., Kleinjans, J. C., and Kogevinas, M. (2012). Birth weight, head circumference, and prenatal exposure to acrylamide from maternal diet: The European prospective motherchild study (NewGeneris). [Article]. Environmental Health Perspectives, 120(12), 1739-1745. doi: 10.1289/ehp.1205327

Pinto, E., Barros, H., and Santos Silva, I. D. (2009). Dietary intake and nutritional adequacy prior to conception and during pregnancy: A follow-up study in the north of Portugal. [Article]. Public Health Nutrition, 12(7), 922-931. doi: 10.1017/S1368980008003595

Rivière, G., Sirot, V., Tard, A., Jean, J., Marchand, P., Veyrand, B., Le Bizec, B., and Leblanc, J. C. (2014). Food risk assessment for perfluoroalkyl acids and brominated flame retardants in the French population: Results from the second French total diet study. [Article]. Science of the Total Environment, 491-492, 176-183. doi: 10.1016/j.scitotenv.2014.01.104

Rothenberg, E. M. (2009). Experience of dietary assessment and validation from three swedish studies in the elderly. [Article]. European Journal of Clinical Nutrition, 63, S64-S68. doi: 10.1038/ejcn.2008.67

Schmidt, M. N., Winther, O., and Kaihansen, L. (2009) Bayesian non-negative matrix factorization. Vol. 5441. Lecture Notes in Computer Science (including subseries Lecture Notes in Artificial Intelligence and Lecture Notes in Bioinformatics) (pp. 540-547).

Sirot, V., Fremy, J.-M., and Leblanc, J.-C. (2013). Dietary exposure to mycotoxins and health risk assessment in the second French total diet study. Food and Chemical Toxicology, 52, 1-11. doi: http://dx.doi.org/10.1016/i.fct.2012.10.036

Sirot, V., Hommet, F., Tard, A., and Leblanc, J. C. (2012a). Dietary acrylamide exposure of the French population: Results of the second French Total Diet Study. [Article]. Food and Chemical Toxicology, 50(3-4), 889-894. doi: 10.1016/j.fct.2011.12.033

Sirot, V., Tard, A., Venisseau, A., Brosseaud, A., Marchand, P., Le Bizec, B., and Leblanc, J. C. (2012b). Dietary exposure to polychlorinated dibenzo-p-dioxins, polychlorinated dibenzofurans and polychlorinated biphenyls of the French population: Results of the second French Total Diet Study. [Article]. Chemosphere, 88(4), 492-500. doi: 10.1016/j.chemosphere.2012.03.004 
Sirot, V., Volatier, J. L., Calamassi-Tran, G., Dubuisson, C., Ménard, C., Dufour, A., and Leblanc, J. C. (2009). Core food of the French food supply: Second Total Diet Study. Food Additives and Contaminants - Part A Chemistry, Analysis, Control, Exposure and Risk Assessment, 26(5), 623-639. doi: 10.1080/02652030802695506

Sly, P. D., and Flack, F. (2008) Susceptibility of children to environmental pollutants. Vol. 1140. Annals of the New York Academy of Sciences (pp. 163-183).

Sy, M. M., Feinberg, M., Verger, P., Barré, T., Clémençon, S., and Crépet, A. (2013). New approach for the assessment of cluster diets. Food and Chemical Toxicology, 52, 180-187. doi: 10.1016/j.fct.2012.11.005

Tan, V. Y. F., and Févotte, C. (2013). Automatic relevance determination in nonnegative matrix factorization with the ( $\beta$ )-divergence. [Article]. IEEE Transactions on Pattern Analysis and Machine Intelligence, 35(7), 1592-1605. doi: 10.1109/TPAMI.2012.240

Team, R. C. (2016). R: A Language and Environment for Statistical Computing (Version 3.3.2): R Foundation for Statistical Computing. Retrieved from https://www.R-project.org/

Traoré, T., Béchaux, C., Sirot, V., and Crépet, A. (2016). To which chemical mixtures is the French population exposed? Mixture identification from the second French Total Diet Study. [Article]. Food and Chemical Toxicology, 98, 179-188. doi: 10.1016/j.fct.2016.10.028

Valvi, D., Mendez, M. A., Martinez, D., Grimalt, J. O., Torrent, M., Sunyer, J., and Vrijheid, M. (2012). Prenatal concentrations of polychlorinated biphenyls, DDE, and DDT and overweight in children: A prospective birth cohort study. Environmental Health Perspectives, 120(3), 451457. doi: 10.1289/ehp.1103862

Vandentorren, S., Bois, C., Pirus, C., Sarter, H., Salines, G., and Leridon, H. (2009). Rationales, design and recruitment for the Elfe longitudinal study. [Article]. BMC Pediatrics, 9, 58. doi: 10.1186/1471-2431-9-58

Veyrand, B., Sirot, V., Durand, S., Pollono, C., Marchand, P., Dervilly-Pinel, G., Tard, A., Leblanc, J.-C., and Le Bizec, B. (2013). Human dietary exposure to polycyclic aromatic hydrocarbons: Results of the second French Total Diet Study. Environment International, 54, 11-17. doi: http://dx.doi.org/10.1016/i.envint.2012.12.011

Wang, G., Kossenkov, A. V., and Ochs, M. F. (2006). LS-NMF: A modified non-negative matrix factorization algorithm utilizing uncertainty estimates. BMC Bioinformatics, 7. doi: 10.1186/1471-2105-7-175

Yang, Z., Zhu, Z., and Oja, E. (2010). Automatic Rank Determination in Projective Nonnegative Matrix Factorization. In V. Vigneron, V. Zarzoso, E. Moreau, R. Gribonval \& E. Vincent (Eds.), Latent Variable Analysis and Signal Separation: 9th International Conference, LVA/ICA 2010, St. Malo, France, September 27-30, 2010. Proceedings (pp. 514-521). Berlin, Heidelberg: Springer Berlin Heidelberg.

Zetlaoui, M., Feinberg, M., Verger, P., and Clémençon, S. (2011). Extraction of food consumption systems by nonnegative matrix factorization (NMF) for the assessment of food choices. Biometrics, 67(4), 1647-1658. doi: 10.1111/j.1541-0420.2011.01588.x 